\title{
Disentangling the Arcturus stream
}

\author{
Iryna Kushniruk and Thomas Bensby
}

\author{
Lund Observatory, Department of Astronomy and Theoretical Physics, Box 43, 22100 Lund, Sweden \\ e-mail: iryna@astro.lu.se, tbensby@astro.lu.se
}

Received 8 February 2019 / Accepted 9 September 2019

\begin{abstract}
Context. The Arcturus stream is an over-density of stars in velocity space and its origin has been much debated recently without any clear conclusion. The (classical) dissolved open cluster origin is essentially refuted; instead the discussions try to distinguish between an accretion, a resonant, or an external-perturbation origin for the stream. As kinematic structures are observational footprints of ongoing and past dynamical processes in disc galaxies, resolving the nature of the Arcturus stream may provide clues to the formation history of the Milky Way and its stellar populations.

Aims. We aim to characterise the kinematical and chemical properties of the Arcturus stream in order to resolve its origin.

Methods. The space velocities, angular momenta, and actions for a sample of more than 5.8 million stars, composed from Gaia DR2 were analysed with a wavelet transform method to characterise kinematic over-densities in the Galactic disc. The kinematic characteristics of each identified group is used to select possible members of the groups from the GALAH and APOGEE spectroscopic surveys to further study and constrain their chemical properties.

Results. In the velocity and angular momentum spaces the already known Sirius, Pleiades, Hyades, Hercules, AF06, Arcturus and KFR08 streams are clearly identified. The Hercules stream appears to be a mixture of thin and thick disc stars. The Arcturus stream, as well as the AF06 and KFR08 streams, are high-velocity and low-angular momentum structures with chemical compositions similar to the thick disc. These three groups extend further from the Galactic plane compared to the Hercules stream. The detections of all the groups were spaced by approximately $20-30 \mathrm{~km} \mathrm{~s}^{-1}$ in azimuthal velocity.

Conclusions. A wide spread of chemical abundances within the Arcturus stream indicates that the group is not a dissolved open cluster. Instead the Arcturus stream, together with the AF06 and KFR08 streams, are more likely to be part of a phase-space wave, that could have been caused by a merger event. This conclusion is based on that the different structures are detected in steps of $20-30 \mathrm{~km} \mathrm{~s}^{-1}$ in azimuthal velocity, that the kinematic and chemical features are different from what is expected for bar-originated structures, and that the higher-velocity streams extend further from the disc than bar-originated structures.
\end{abstract}

Key words. stars: kinematics and dynamics - Galaxy: formation - galaxies: kinematics and dynamics - galaxies: evolution

\section{Introduction}

The process by which large spiral galaxies form and evolve into the complicated structures that are observed today is an active area of research, and presents many challenges, both theoretically and observationally. As the Milky Way is the only galaxy where stars and structures can be studied in great detail, it serves as a benchmark galaxy when constraining models of galaxy formation. It is therefore of utmost importance to obtain a detailed map of the Milky Way looks like, and to decipher where the observed stellar populations and structures come from. Currently, the Milky Way contains a plethora of structures, both physical and kinematic, whose nature and origins are unclear.

Many studies have shown that the velocity distribution of stars in the Milky Way disc is clumpy (e.g. Dehnen 1998; Skuljan et al. 1999; Famaey et al. 2005; Antoja et al. 2008, 2012; Kushniruk et al. 2017; Ramos et al. 2018). The kinematic and chemical properties of such structures can be used to constrain the properties and the formation history of the Milky Way. For example, the Hercules stream has been widely used to probe the pattern speed and the length of the Galactic bar (e.g. Dehnen 2000; Minchev et al. 2007; Antoja et al. 2014; Wegg et al. 2015; Pérez-Villegas et al. 2017). Kinematic structures can be used to study the spiral structure of the Milky Way (e.g. Quillen \& Minchev 2005; Chakrabarty 2007; Sellwood et al. 2019; Quillen et al. 2018). Studies of kinematic streams especially in the Galactic halo can tell us about the merger history of the Milky Way (e.g. Navarro et al. 2004; Helmi et al. 2006, 2017, 2018; Koppelman et al. 2018). The analysis of the Gaia DR2 (Gaia Collaboration 2018a,b) revealed that the kinematic over-densities are a part of a much more complicated structure that is seen as arches and ridges across velocity space and as clumps in action space (Trick et al. 2019). This structure is possibly caused by spiral arms (Quillen et al. 2018) or is a result of the phase-mixing due to a past merger event (e.g. Katz et al. 2019; Antoja et al. 2018; Ramos et al. 2018; Laporte et al. 2019), as first proposed by Minchev et al. (2009).

This structure is possibly caused by spiral arms or is a result of phase-mixing due to a past merger event (e.g. Antoja et al. 2018; Ramos et al. 2018; Quillen et al. 2018). As these studies have shown, learning more about the nature of kinematic structures can improve our understanding of the evolution of the Milky Way. In this paper we investigate the properties and origin of the Arcturus stream.

A set of about 50 stars, including the star Arcturus ( $\alpha$ Bootis), was discovered by Eggen (1971) to have a very similar V space velocity component of $V \simeq-100 \mathrm{~km} \mathrm{~s}^{-1}$. Eggen (1971) proposed that this over-density in velocity space is composed of stars that escaped from an open cluster and it was therefore named the Arcturus moving group. Nowadays the hypothesis of the Arcturus over-density being a moving group is refuted as there is no chemical homogeneity within the group (e.g. Williams et al. 2009; Ramya et al. 2012; Bensby et al. 2014), 
which there should be if the stars originate from the same open cluster (e.g. De Silva et al. 2007; Bovy 2016). We have therefore chosen to adopt the "stream" nomenclature when referring to this Arcturus over-density of stars in velocity space.

Two other possible origins of the Arcturus stream are now favoured and are widely discussed. The first is an accretion event scenario, where a small satellite galaxy merged with the Milky Way and caused this dynamical structure (e.g. Navarro et al. 2004; Helmi et al. 2006; Minchev et al. 2009). The second possibility is that it has originated due to resonances with the Galactic bar or spiral arms that cause kinematic over-densities (e.g. Gardner \& Flynn 2010; Monari et al. 2013). The chemical properties of the stream do not show any chemical peculiarities, which would be expected in the case of an extra-Galactic origin (e.g. Ramya et al. 2012; Bensby et al. 2014). At the same time the low angular momentum and the low velocity of the stream indicate that it could be another substructure of tidal debris in the Galactic halo (e.g. Arifyanto \& Fuchs 2006; Klement et al. 2008; Zhao et al. 2014). Despite numerous approaches to study the origin of the Arcturus stream (e.g., numerical simulations, kinematic analysis, and studies of elemental abundances), there is no consensus on its origin.

The aim of this paper is to characterise the nature of the Arcturus stream and constrain its origin. We start by detecting and characterising the velocities of the Arcturus stream using a large stellar sample constructed from the Gaia DR2 catalogue (see Sect. 2). We then search for over-densities in the velocity, angular momentum, and action spaces to obtain the kinematic characteristics of the stream (see Sects. 3-5). Subsequently, we investigate the chemical characteristics of the group using the data from the GALAH (Buder et al. 2018) and APOGEE (Holtzman et al. 2018) spectroscopic surveys (see Sect. 6). We conclude by discussing the possible origins for the Arcturus stream based on the kinematic and spectroscopic findings (see Sects. 7 and 8).

\section{Stellar sample}

To search for the Arcturus stream, a wavelet analysis was applied for a stellar sample defined by velocities, angular momentum, and action components. To calculate these parameters positions on the sky, proper motions, parallaxes, radial velocities, and the corresponding uncertainties for these properties are needed.

The size of the stellar sample and the quality of the astrometric data play a key role when hunting for kinematic structures. As the size of the samples of stars with available high-precision astrometric data increases, so does the level of detail with which it is possible to study kinematical structures of the Galaxy. The currently best data source is the Gaia satellite, which is an ongoing full-sky mission that aims to provide high-precision astrometric parameters for more than a billion targets over the whole sky. The most recent data release, Gaia DR2 (Gaia Collaboration 2018a), contains astrometric data for almost 1.7 billion targets, and radial velocities for a small subsample of about 7 million targets.

A stellar sample of 5844487 stars was constructed from the Gaia DR2 catalogue in the following way:

- 7173615 stars were obtained from McMillan (2018), who estimated distances for Gaia DR2 stars with measured radial velocities.

- Stars with bad fits of Gaia DR2 astrometric parameters were filtered out to avoid possible systematic errors in the stellar sample. Following the procedure suggested in Lindegren (2018) a re-normalised unit weight error (RUWE) was used to estimate goodness of astrometric fits. Selecting those targets with RUWE < 1.4 leaves us with 6692285 targets. Photometric filtering that rids the sample of stars with poor astrometric solutions (see Eq. (2) in Arenou et al. (2018)) was also applied. This cut leaves us with 6683408 stars.

- Space velocities $U, V, W^{1}$ together with angular momenta and actions that are used below were computed using the galpy ${ }^{2}$ package (Bovy 2015). For action estimates we used a MWPotential2014 axisymmetric gravitational potential model pre-defined in galpy. Velocity uncertainties $\sigma_{U}, \sigma_{V}, \sigma_{W}$ were computed following equations from Johnson \& Soderblom (1987). The velocities are given relative to the Local Standard of Rest: $\left(U_{\odot}, V_{\odot}\right.$, $\left.W_{\odot}\right)=(11.1,12.24,7.25) \mathrm{km} \mathrm{s}^{-1}$ (Schönrich et al. 2010).

Taking into account the results from, for example, Zhao et al. (2014), the typical size of kinematical structures is around $20 \mathrm{~km} \mathrm{~s}^{-1}$. Therefore we need to cut stars with $\sigma_{U}, \sigma_{V}>20 \mathrm{~km} \mathrm{~s}^{-1}$, because such large velocity uncertainties will influence the precision of the results, that is the position in velocity space of the structures. This leaves us with 6002514 stars.

- Next, the sample was constrained to stars that are located within a distance of $5 \mathrm{kpc}$ from the Sun. This filters out stars that are located in the outskirts or in the very inner parts of the Galaxy, and thus, cannot be a part of any of the local kinematic structures. The limit of $5 \mathrm{kpc}$ was chosen to avoid regions in direct contact with for example the Galactic bar, whose halflength is about $3 \mathrm{kpc}$ (e.g. Dehnen 2000; Minchev et al. 2010; Monari et al. 2017). According to Bailer-Jones (2015), distance estimates should not be dominated by using pre-Gaia information or so-called priors if fractional parallax uncertainty does not exceed $20 \%$. Typical parallax uncertainty for bright sources in Gaia DR2 is about $0.4 \mu$ as (Lindegren 2018). Converting the $5 \mathrm{kpc}$ cut into $\mu$ as and calculating fractional parallax uncertainty we obtain $20 \%$ meaning that distance estimates in the sample should not be effected by priors. This cut leaves 5844487 that are used in our analysis.

Since kinematic structures are local phenomena (e.g. Antoja et al. 2012; Ramos et al. 2018; Trick et al. 2019) and the stellar sample covers a wide range in $X$ and $Y$, it was divided into 66 smaller volumes that were investigated separately. Each box is $0.4 \mathrm{kpc}$ in radial coordinates and $3^{\circ}$ in azimuthal angle ${ }^{3}$. The top plot in Fig. 1 shows the distribution of the 5844487 stars in the Galactic Cartesian $X-Y$ plane and how it is divided into small volumes. The name of each region, the number of stars, the median distance from the Sun, and median distance uncertainty are given in Table A.1 for each of the 65 volumes. The bottom plot in Fig. 1 shows the sample in Cartesian $X$ and $Z$ coordinates, where $Z$ is a vertical component of Galactocentric coordinate system (points towards the north Galactic pole).

\section{Method}

Our current knowledge of the Arcturus stream is based on observations within a small region of about $500 \mathrm{pc}$ around the Sun. Its origin is unknown, mainly due to the fact that there are only rough estimates of its kinematic characteristics and its chemical properties are not well studied. Our strategy is therefore to search for streams in four different planes defined by

\footnotetext{
$1 U$ points towards Galactic centre, $V$ velocity defines the direction of the Galactic rotation, $W$ points at the North Galactic Pole.

2 Available at http://github. com/jobovy/galpy

$3 R$ is the radial coordinate pointing towards the Galactic anti-centre, and $\phi$ is the azimuthal angle following the direction opposite to the
} Galactic rotation. 

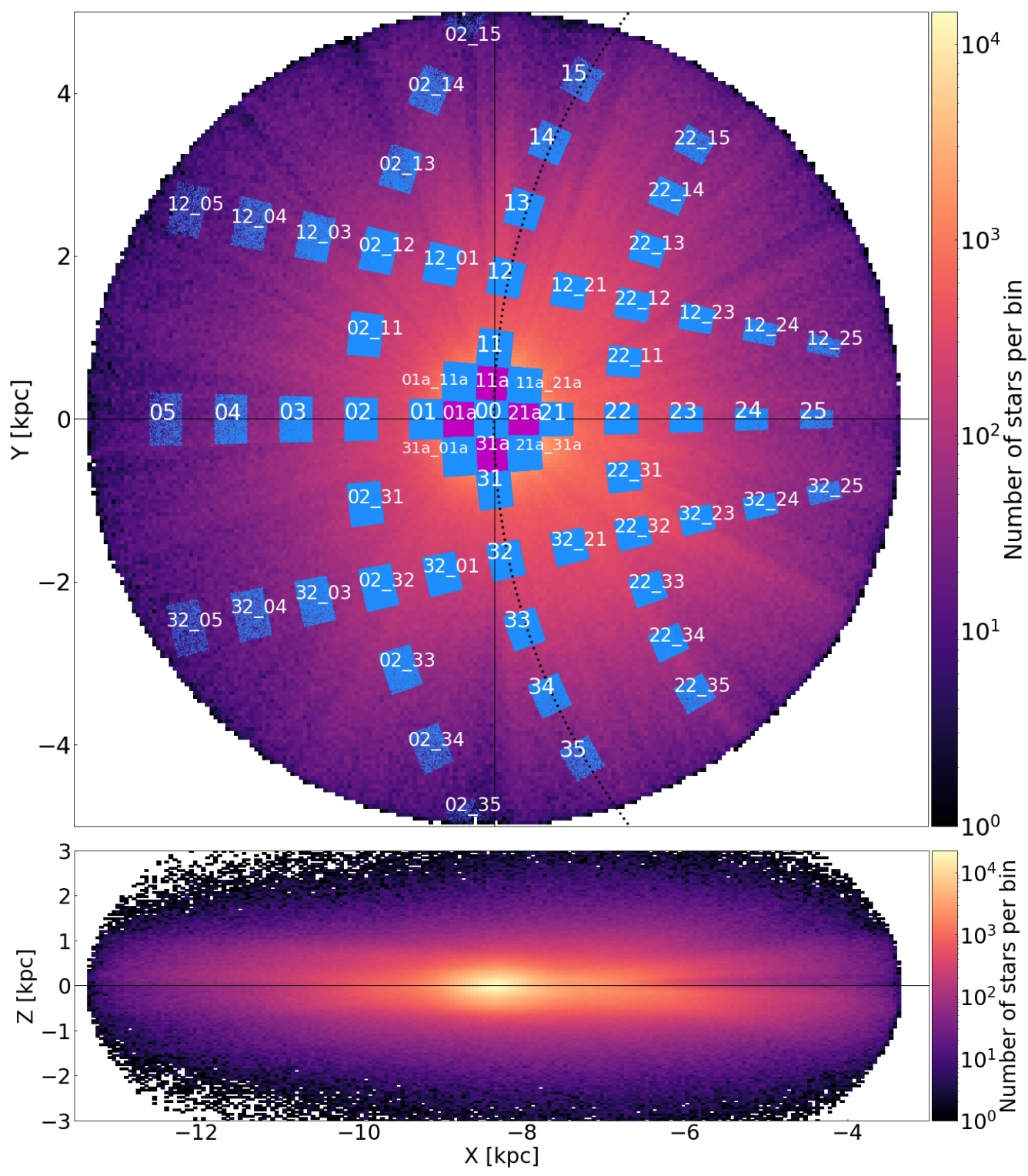

Fig. 1. Distribution of 5844487 stars with $\sigma_{U}$ and $\sigma_{V}<20 \mathrm{~km} \mathrm{~s}^{-1}$ and $R \leq 5 \mathrm{kpc}$ in $X$ and $Y$ (top), and $X$ and $Z$ (bottom) Cartesian Galactic coordinates. Blue and magenta boxes show 66 small regions investigated in this work and their names are written at the top in white. The dashed line in the top plot shows the solar circle. The black line in the bottom plot shows $Z=0 \mathrm{kpc}$. The bin size is $0.05 \mathrm{kpc}$ for both plots.

combinations of velocity, angular momentum and action components respectively: the $U-V$ plane, the $V-\sqrt{U^{2}+2 V^{2}}$ plane, the $L_{z}-\sqrt{L_{x}^{2}+L_{y}^{2}}$ plane, and the $L_{z}-\sqrt{J_{\mathrm{r}}}$ plane. This will allows to characterise structures in terms of velocities, angular momenta, and actions, and obtain stronger criteria on how to select star members of kinematic structures.

\subsection{Investigated planes}

The distributions of stars in all four planes are shown in Fig. 2. The majority of the stars in our sample have negative $V$ velocities between $V \simeq 0$ and $-200 \mathrm{~km} \mathrm{~s}^{-1}$ and angular momentum $L_{z}$ between 0 and $2500 \mathrm{kpc} \mathrm{km} \mathrm{s}^{-1}$. The disc stars are located at $L_{z} \simeq 1800 \mathrm{kpc} \mathrm{km} \mathrm{s}^{-1}$, the halo stars are expected at $L_{z} \simeq 0 \mathrm{kpc} \mathrm{km} \mathrm{s}^{-1}$.

\subsubsection{The $U-V$ plane}

The $U-V$ plane is widely used to search for kinematic structures (e.g. Dehnen 1998; Antoja et al. 2008, 2012, 2018; Kushniruk et al. 2017; Ramos et al. 2018; Katz et al. 2019). It allows to trace kinematic over-densities of different origin without making any assumptions on orbital parameters of stars or on the Galactic potential. The only limitation of this method is that stellar volumes must be relatively small (around $0.1-0.5 \mathrm{pc}$ in $X$ and $Y$; see Trick et al. 2019), since kinematic structures in the $U-V$ plane are local. On the other hand, this limitation is an advantage, since it allows us to follow how the structures move in physical space (Ramos et al. 2018). The Arcturus stream is expected to be one of the arches in the $U-V$ plane localised around $V \simeq-100 \mathrm{~km} \mathrm{~s}^{-1}$ in the nearby sample. The stream is likely to cover a wide range of $U$ velocities (Williams et al. 2009). 

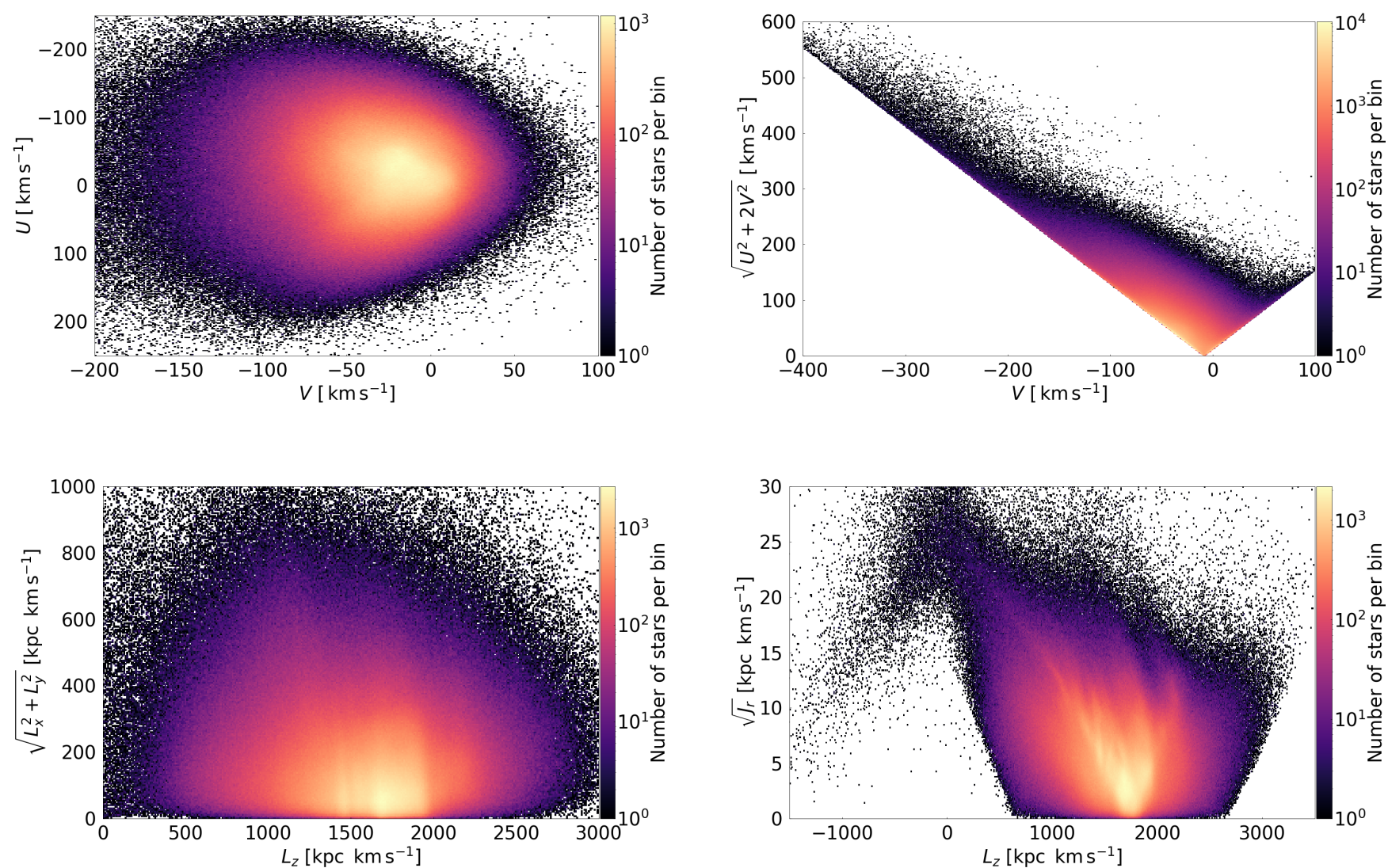

Fig. 2. Density map of the selected sample of 5844487 stars in $U-V$ space (top left), in $V-\sqrt{U^{2}+2 V^{2}}$ space (top right), in $L_{z}-\sqrt{L_{x}^{2}+L_{y}^{2}}$ space (bottom left), and in $L_{z}-\sqrt{J_{\mathrm{R}}}$ space (bottom right). The bin sizes are $1 \mathrm{~km} \mathrm{~s}^{-1}, 1 \mathrm{~km} \mathrm{~s}^{-1}, 10 \mathrm{kpc} \mathrm{km} \mathrm{s}{ }^{-1}$, and $10 \times 0.1 \mathrm{kpc} \mathrm{km} \mathrm{s}{ }^{-1}$, respectively.

\subsubsection{The $V-\sqrt{U^{2}+2 V^{2}}$ plane}

Examining the distribution of stars in the $V-\sqrt{U^{2}+2 V^{2}}$ plane was proposed by Arifyanto \& Fuchs (2006). Here, $V$ is proportional to $L_{z}$, a vertical component of the angular momentum, and is an integral of motion in axisymmetric potentials. $\sqrt{U^{2}+2 V^{2}}$ is a measure of eccentricity in Dekker's approximation (Dekker 1976). This means that we search for structures that share similar orbital eccentricity. This approach is applicable only for planar orbits in axisymmetric potentials. The method shows reliable results for nearby stars with eccentricities up to 0.5 (Arifyanto \& Fuchs 2006). The $V-\sqrt{U^{2}+2 V^{2}}$ plane was used by Klement et al. (2008) and Zhao et al. (2014) to search for kinematic structures and allowed to reveal several structures including the Arcturus stream. We expect to detect the Arcturus stream at the velocities around $V \simeq-100 \mathrm{~km} \mathrm{~s}^{-1}$ in the solar neighbourhood (e.g. Williams et al. 2009).

\subsubsection{The $L_{z}-\sqrt{L_{x}^{2}+L_{y}^{2}}$ plane}

Another approach to search for kinematic groups was proposed by Helmi et al. (1999) who suggested to examine the distribution of stars in the plane characterised by the $L_{z}$ and $\sqrt{L_{x}^{2}+L_{y}^{2}}$ integrals of motion, where $L_{x}, L_{y}$, and $L_{z}$ are angular momentum components in $X, Y$, and $Z$ directions. The method is used to search for phase-mixed stars on similar orbits. The disadvantage of this method is that $\sqrt{L_{x}^{2}+L_{y}^{2}}$ is not fully conserved in axisymmetric potentials, but still allows dynamical structures to be revealed (e.g. Helmi et al. 1999; Klement et al. 2008; Zhao et al. 2014). The Arcturus stream is expected at $L_{z}$ in the range between 700 and $1100 \mathrm{~km} \mathrm{~s}^{-1} \mathrm{kpc}$ (e.g. Navarro et al. 2004).

\subsubsection{The $L_{z}-\sqrt{J_{\mathrm{r}}}$ plane}

The most general method to search for kinematic structures is to investigate action space. Actions are conserved quantities that characterise stellar orbits. In this work we use radial and azimuthal actions $J_{\mathrm{r}}$ and $L_{z}$ that are a measure of orbital eccentricity and orbital angular momentum. As suggested in Trick et al. (2019) taking the square root of radial action will make the final plots more clear. The action space was investigated by, for example, Sellwood (2010) and Trick et al. (2019) and is rich in kinematic over-densities. We expect to detect the Arcturus stream at $L_{z}$ in the range between 700 and $1100 \mathrm{kpc} \mathrm{km} \mathrm{s}^{-1}$ (e.g. Navarro et al. 2004).

\subsection{Wavelet transform}

To search for kinematic structures, the methodology described in Kushniruk et al. (2017) was used with some additions. To detect over-densities, a wavelet transform was applied to the stellar sample in the $U-V, V-\sqrt{U^{2}+2 V^{2}}, L_{z}-\sqrt{L_{x}^{2}+L_{y}^{2}}$, and $L_{z}-\sqrt{J_{\mathrm{r}}}$ planes. The noise from the wavelet maps was then filtered and Monte Carlo simulations were used to verify whether the detected structures are real or not. 
The data were analysed by the wavelet transform with the "a trous" algorithm (Starck \& Murtagh 1998) applied to the stars in all 65 regions in the four different planes separately. The input data are in the form of a binned stellar density map in the velocity, angular momentum, and action planes. The bin sizes $\Delta$ were set to $1 \mathrm{~km} \mathrm{~s}^{-1}$ for the $U-V$, and the $V-\sqrt{U^{2}+2 V^{2}}$ planes, to $2 \mathrm{~km} \mathrm{~s}^{-1} \mathrm{kpc}$ for the $\sqrt{L_{x}^{2}+L_{y}^{2}}-L_{z}$ plane, and to $0.1 \times$ $10 \mathrm{kpc} \mathrm{km} \mathrm{s}^{-1}$ for the $L_{z}-\sqrt{J_{\mathrm{r}}}$ plane. Due to the limitations of the usage of the $V-\sqrt{U^{2}+2 V^{2}}$ plane, as discussed in Sect. 3.1.2, the stars that have orbits with eccentricities $e>0.5$ were cut out. The output data are a set of wavelet coefficients at different scales that contain information about the presence of substructures. A higher wavelet coefficient means a higher probability that the structure is real. The scale $J$ is proportional to the size of the detectable structures $s$. Scales $J=1,2,3$ and 4 were investigated for all maps. The relation between scale and bin size $s_{J}=2^{J} \Delta$ characterises the typical sizes of detectable structures. The wavelet coefficient maps were then filtered for Poisson noise. The wavelet transform part as well as noise filtering from the output wavelet maps were performed in The multiresolution analysis software (MR software) ${ }^{4}$ developed by CEA (Saclay, France) and the Nice Observatory. More details on the algorithm itself can be found in Starck \& Murtagh (2002), and more details on the methods used to search for over-densities and structures can be found in Kushniruk et al. (2017).

\subsection{Acquiring positions of the detected peaks}

Monte Carlo (MC) simulations were performed to obtain the precise positions of the peaks. Monte Carlo samples were created assuming that each star can be represented as a Gaussian velocity distribution with $\mu=(U, V)$ and $\sigma=\left(\sigma_{U}, \sigma_{V}\right)$ for the two velocity components. To generate MC samples in angular momentum and action space, the orbits of stars were computed assuming that positions, proper motions, and radial velocities can be represented as Gaussians in a similar way to velocities. Here it is assumed that Gaussians are independent and do not consider correlations between astrometric parameters. These MC samples are then analysed in the same manner as the original data. Convergence is reached when the number of structures and their positions do not change as more simulations are added. Typically, results converge after about 30 simulations, but to be sure of convergence, $100 \mathrm{MC}$ samples were created for all regions. Subsequently, MC wavelet maps for different scales were over-plotted and used to search for peaks by applying the peak_local_max feature from the scikit-image 5 Python package (van der Walt et al. 2014). In this work we focus on the $J=2$ and $J=3$ scales as they allow us to detect most structures.

\section{Results}

\subsection{Stellar streams in the nearby sample}

Figures 3 and 4 show 100 over-plotted wavelet maps for the central region 00 in four different planes for scales 2 and 3, respectively. Both scales show richness of kinematic structures for the nearby sample. The list of the centres of the peaks and the corresponding uncertainties are given in Table A. 2 for scale $J=2$ and in Table A. 3 for scale $J=3$. The fact that well-known groups like Sirius, Coma Berenices, Hyades, Pleiades, and Hercules

\footnotetext{
4 Available at http://www . multiresolutions. com $/ \mathrm{mr} /$

5 https://scikit-image.org/
}

were identified at the expected positions shows that our method is sound (see Tables 3-7 in Kushniruk et al. 2017 that summarise literature values for the $U$ and $V$ velocities of Sirius, Coma Berenices, Hyades, Pleiades, and Hercules). These groups are detected in all four planes. The detection of other groups varies between the planes. Figure 3 for scale $J=2$ shows the same structures as in Fig. 4 for scale $J=3$ but in greater detail. We decided to focus on scale $J=2$ since it is more sensitive to smaller structures. By comparing our results in the nearby region " 00 " (centred around the Sun; see Fig. 2) for scale $J=2$ with what has previously been found in the literature (e.g. Eggen 1998; Navarro et al. 2004; Arifyanto \& Fuchs 2006; Klement et al. 2008; Williams et al. 2009; Antoja et al. 2012, 2018; Zhao et al. 2014; Kushniruk et al. 2017; Trick et al. 2019; Ramos et al. 2018) we assign names to the structures. Curved lines and boxes of different colours in Figs. 3 and 4 correspond to the names of the groups listed in the legend of Fig. 3. The structures found in the nearby region are discussed below:

- A1/A2: Groups with $V>15 \mathrm{~km} \mathrm{~s}^{-1}$ and $L_{z}>2000 \mathrm{kpc}$ $\mathrm{km} \mathrm{s}^{-1}$ we link to arches A1 and A2 detected by Ramos et al. (2018, see their Table 2). Groups A1 and A2 is shown with yellow lines and boxes on the plots.

- Sirius: The blue line and boxes correspond to the Sirius stream. Group 13 in the $U-V$ space is potentially Bobylev16 (see Bobylev \& Bajkova 2016) and could be a continuation of Sirius.

- $\gamma$ Leo: The pink line slightly above Sirius in $V$ is $\gamma$ Leo stream (see Antoja et al. 2012). Unlike the majority of the groups, $\gamma$ Leo is located at positive $U$ velocities. The stream could be a continuation of Sirius arch since both have similar angular momenta.

- Coma Berenices: The magenta line just below Sirius is Coma Berenices stream. Unlike arch-like neighbouring Sirius and Pleiades/Hyades, Coma Berenices is a clump in the $U-V$ plane and is consequently a shorter line in the angular momentum space.

- Dehnen98/Wolf630: Wolf630 and Dehnen98 (see Antoja et al. 2012; Dehnen 1998) are two small groups in between Coma Berenices and Pleiades/Hyades streams. They are shown in brown colour and could be a continuation of Coma Berenices.

- Pleiades/Hyades: A grey arch in the $U-V$ plane is associated with the Pleiades/Hyades stream. Group 29 in the $U-V$ space linked to Antoja12(15) (see Antoja et al. 2012) could be a continuation of stream.

- Hercules: Orange lines and boxes correspond to the Hercules stream, which is likely to be composed of a few substructures that are visible in the angular momenta and action spaces.

- HR1614: The HR1614 moving group (see Feltzing \& Holmberg 2000; De Silva et al. 2007) we connect to the clumps just below Hercules in $V$. The group is shown in lime green.

$-\epsilon$ Ind: Groups g34 and g35 in the $U-V$ plane are linked to a group called $\epsilon$ Ind (see Antoja et al. 2012). The structure is marked in black.

- AF06: The AF06 stream was first found by Arifyanto \& Fuchs (2006) in the range between $V \simeq-70$ and $-100 \mathrm{~km} \mathrm{~s}^{-1}$. We did not find it in the $U-V$ space, but the group is detected in the other three spaces and is shown with red boxes.

- Arcturus: Group g36 in the $U-V$ plane could be the Arcturus stream. Median $V$ velocity and angular momentum of $\mathrm{g} 36$ are $V \simeq-92 \mathrm{~km} \mathrm{~s}^{-1}$ and $L_{z} \sim 1118 \mathrm{kpc} \mathrm{km} \mathrm{s}^{-1}$. These values are a bit higher compared to for example values from Navarro et al. (2004), but are within the uncertainties. In the $V-\sqrt{U^{2}+2 V^{2}}$ plane the nearest to Arcturus are groups g1 and g2 which have 

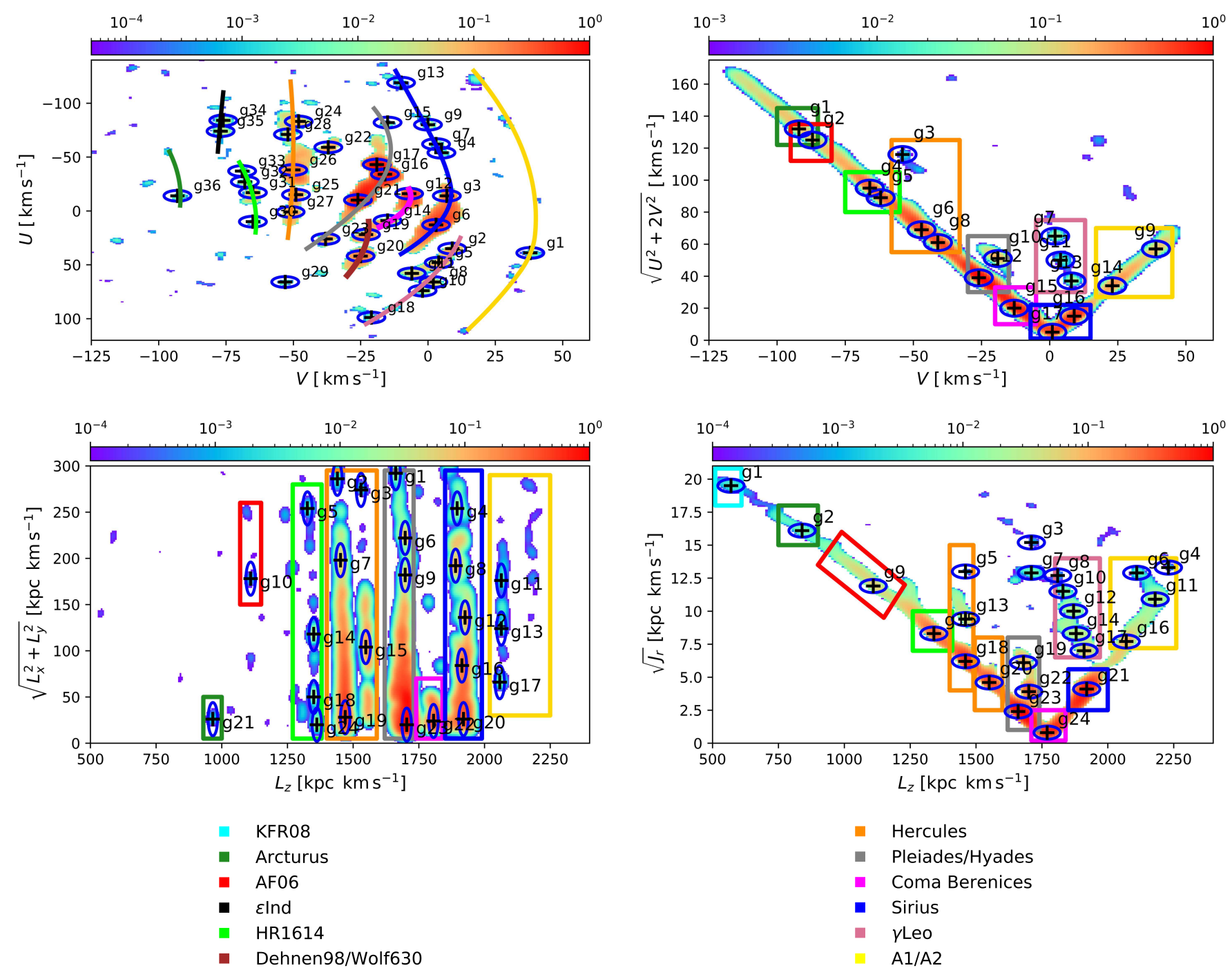

Fig. 3. Wavelet coefficient maps of central region 00 retrieved in $U-V$ (top left), $V-\sqrt{U^{2}+2 V^{2}}$ (top right), $L_{z}-\sqrt{L_{x}^{2}+L_{y}^{2}}$ (bottom left), and $L_{z}-\sqrt{J_{\mathrm{R}}}$ (bottom right) space for scale $J=2$. Colour bars show normalised wavelet coefficients. Kinematic structures are shown as blue circles with radius $5 \mathrm{~km} \mathrm{~s}^{-1}$ or $5 \mathrm{kpc} \mathrm{km} \mathrm{s}^{-1}$ and their centres are shown with black crosses. Lines and boxes of different colours correspond to the group names as listed in the legend. We note that numbers assigned to the groups do not match between the planes.

the same angular momentum and radial action as $\mathrm{g} 36$ in the $U-V$ plane. In the angular momentum space g21 has the parameters closest to Arcturus. In action space there are two candidates: g9 and g2. The first group is consistent with the groups detected in velocity spaces, the second one has lower angular momentum and higher $V$ velocity. Taking into account works by for example Klement et al. (2008) and Zhao et al. (2014) we link g2 to the Arcturus stream and group g9 to the AF06 stream. Arcturus is shown as green lines and boxes on the wavelet maps.

- KFR08: Among the detected groups we assign one weak over-density in action space at $L_{z} \simeq 575 \mathrm{kpc} \mathrm{km} \mathrm{s}^{-1}$ to the group called KFR08. The structure was first detected by Klement et al. (2008) at $V \simeq-160 \mathrm{kpc} \mathrm{km} \mathrm{s}^{-1}$. Group g1 detected in action space has exactly the same median $V$ velocity. KFR08 is shown in cyan on the wavelet maps.

Overall, 36 groups at scale $J=2$ and 16 groups at scale $J=3$ were discovered in the $U-V$ plane that form largerscale arches as discussed in Ramos et al. (2018), Antoja et al. (2018), and Gaia Collaboration (2018b). We also conclude that these arches correspond to the lines in the $L_{z}-\sqrt{L_{x}^{2}+L_{y}^{2}}$ plane and to clumps in the $V-\sqrt{U^{2}+2 V^{2}}$ and $L_{z}-\sqrt{J_{\mathrm{r}}}$ due to the very similar properties of the groups (see Tables A.2 and A.3). In the $V-\sqrt{U^{2}+2 V^{2}}, 17$ and 8 groups were detected. Action space very closely mimics the $V-\sqrt{U^{2}+2 V^{2}}$ plane very much but allows the structures to be detected in greater detail. In the angular momentum and action spaces, 24 groups were found in each space at scale $J=2$ and 9 and 10 groups at scale $J=3$, respectively.

\subsection{Stellar streams outside the solar neighbourhood}

The solar neighbourhood volume is well-studied and thus it is relatively easy to match the detected groups with groups that have been identified by other studies in the literature. The behaviour of the groups outside the solar volume was studied by for example Antoja et al. (2012) and Ramos et al. (2018). Both of these latter studies found a decreasing trend for $V$ velocity when moving to the volumes at larger $R$. In this work we also investigated the trends of the structures depending on the position in the Galaxy with a focus on the Arcturus stream. 

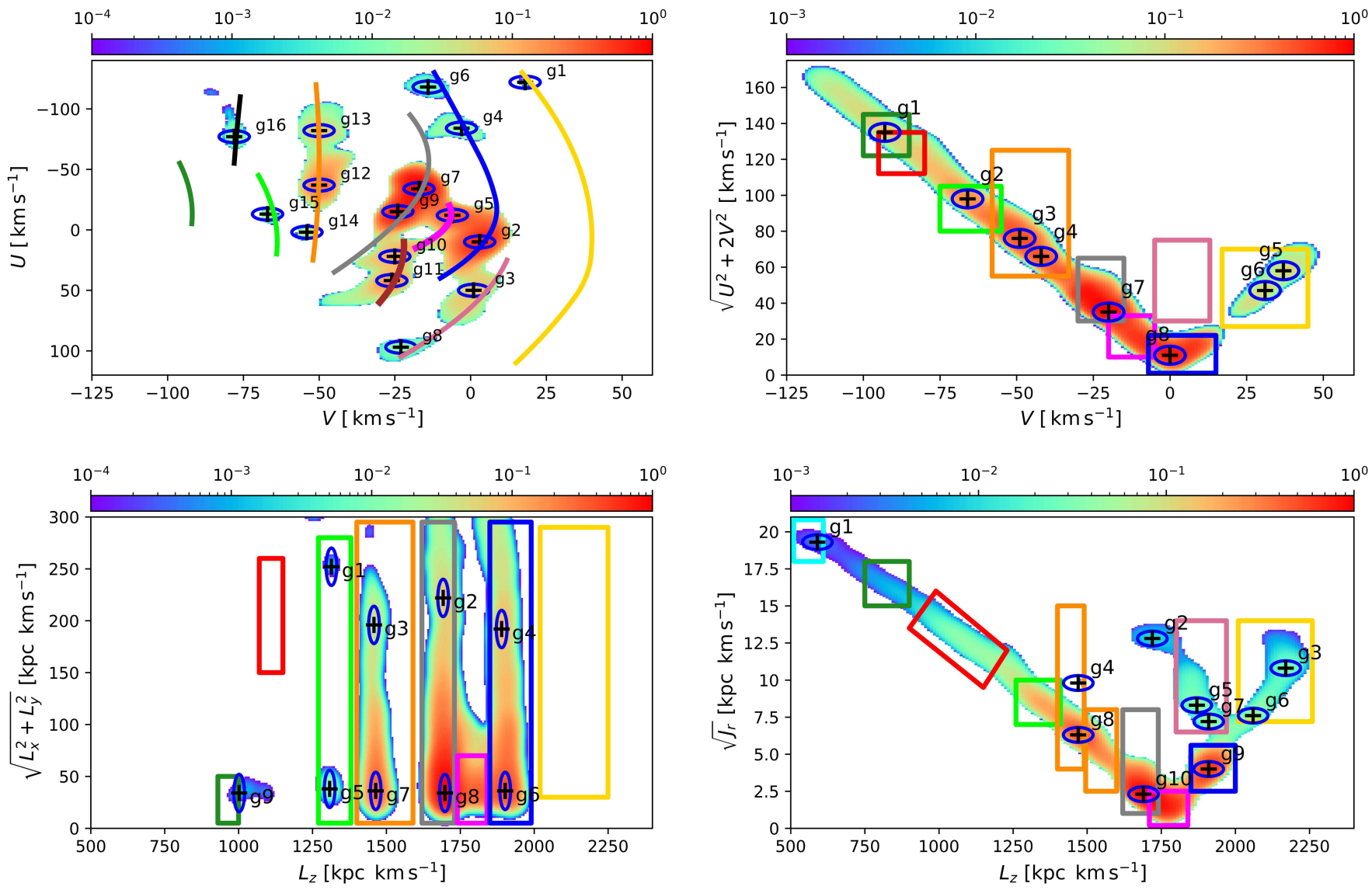

Fig. 4. Same as Fig. 3 but for scale $J=3$. Lines and boxes correspond to the structures detected at scale $J=2$. We note that numbers assigned to the groups do not match between the planes.

If one looks at the small volumes from the left-hand side of Fig. 2 and move towards positive $X$ values an increase in $V$ velocity can be observed. As an example of this trend, Fig. 5 shows how the $V$ velocity of the groups evolves with Galactocentric distance $R$ in regions $01,01 \mathrm{a}, 00,21$, and 21 a based on the analysis of the $U-V$ plane. For example, the Hercules stream in region 01 is detected at $V \simeq-70 \mathrm{~km} \mathrm{~s}^{-1}$, and for comparison, in region 21a the Hercules stream shifts towards positive $V$ and is located at $V \simeq-40 \mathrm{~km} \mathrm{~s}^{-1}$. Similar behaviours are observed for most of the major streams and is shown in the top plot of Fig. 5. On the wavelet maps for regions 01a and 21a that are shown in the middle and bottom plots of Fig. 5 we draw the lines from the top left plot in Fig. 3. Taking volumes at larger $R$ shifts the groups towards higher $V$ values and vice verse.

If one fixes the Galactocentric distance and starts exploring the regions at high $\phi$ moving down towards negative $\phi$ (e.g., start at region 15 and go down to region 35), the streams are observed at the same position in $V$. Major streams including Sirius, Pleiades, Hyades, and Hercules have the same angular momentum when fixing $R$ and looking at different $\phi$. This is different from results in Monari et al. (2019) who found that the Hercules angular momentum changes with azimuth at solar radius. We also do not observe this change when fixing $R$ inside and outside the solar circle.

The shape of the angular momentum and action spaces changes slightly at different $R$, but almost all main structures detected in the solar volume remain at the same positions within a box defined by volumes 02_12, 22_12, 22_32 and 02_32. Figure 6 shows wavelet transform maps for regions 01a and 21a (top and middle rows). Additionally a wavelet transform was applied to all the stars in the sample in the angular momentum and action spaces (bottom row) and was compared to results in volumes $01 \mathrm{a}$ and $21 \mathrm{a}$. If there are any groups with constant actions detected in the total sample then it possible to observe them in smaller volumes. Boxes of different colours mark kinematic structures detected in the region 00 . The same boxes are plotted on top of maps for regions 01a and 21a. There is a small shift in action space when changing $R$, but generally main groups are located at the same positions.

The kinematic structures are mainly detected in the central regions within the rectangle defined by regions $02 \_12,22 \_12$, 22_32 and 02_32. The rest of the remaining regions contain less stars and also have larger distance uncertainties. We tested whether or not the structures really exist only inside the box mentioned above or whether or the lack of the groups in the outer regions is a consequence of larger distances and smaller numbers of stars in subsamples. To investigate the latter, 10000 stars were randomly selected in the central region 00 and the wavelet analysis was repeated. The results were then compared with Dehnen (1998) who used a sample of 14000 stars in total. For our 10000 sample, a similar result to that of Dehnen (1998) was received. The main conclusion of the test is that with the small samples it is possible to detect only the large main structures like Sirius, Hyades, Pleiades and Hercules. The more stars are in the volume, the higher the probability of detecting highvelocity structures. Due to this limitation, it is not possible claim that there is such a radius where some of the groups stop existing. 

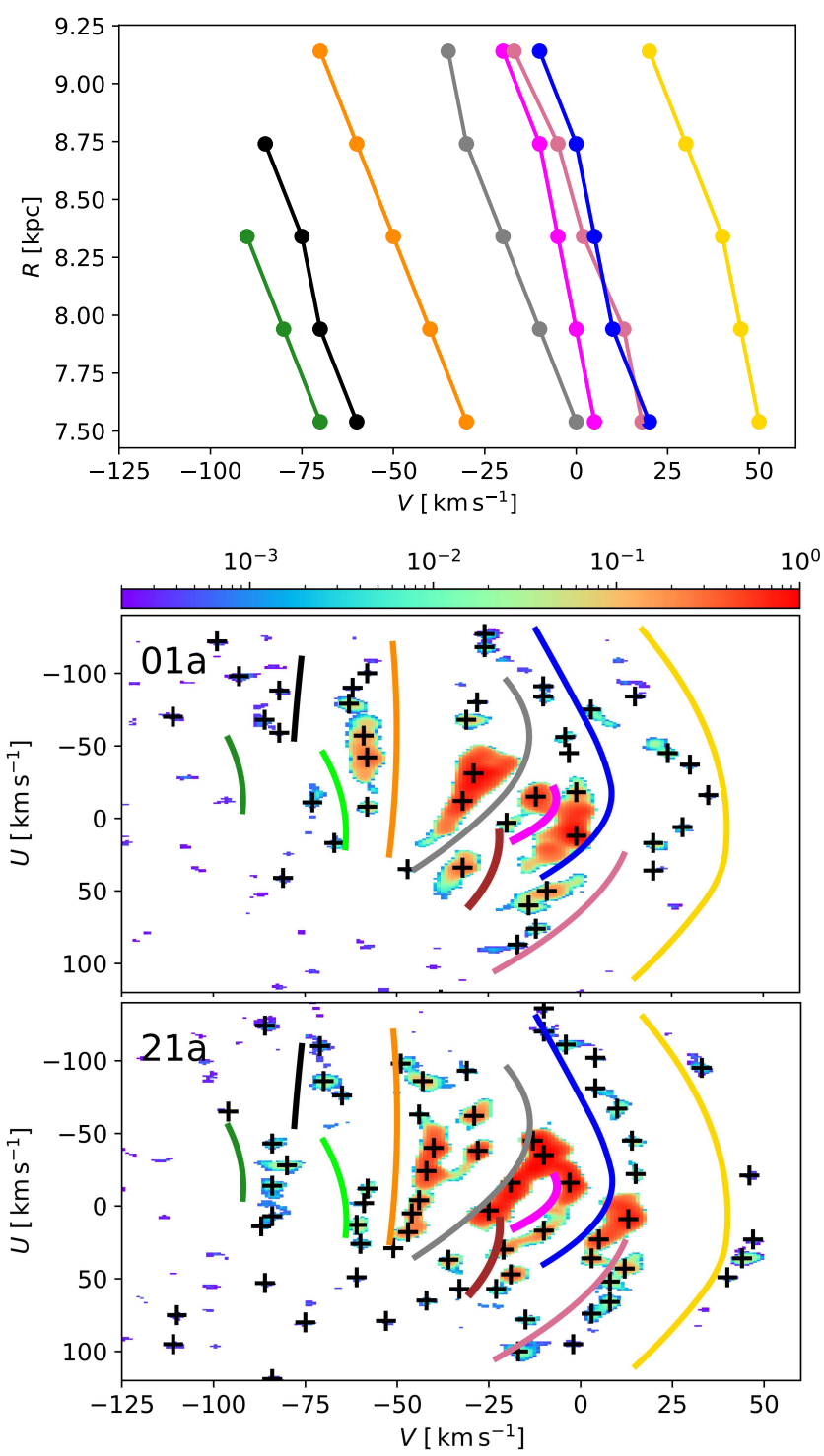

Fig. 5. Top: change of central $V$ velocities of kinematic structures as a function of Galactocentric radii $R$. Each dot corresponds to the centre of the structure determined by the wavelet transform in volumes 01, 01a, 00, 21, and 21a. Names of the groups associated with the lines are listed in the legend of Fig. 3. Middle and bottom: example of wavelet maps in the $U-V$ space in regions 01a and 21a. Colour bar shows normalised wavelet coefficients. Black crosses show centres of the detected structures in regions 01a and 21a. Lines show the location of kinematic structures detected in region 00 (see legend of Fig. 3).

There are many tiny groups detected in the $U-V$ plane that could potentially be a part of the Arcturus stream. We do not observe a clear arch that we can connect with the structure. Unlike the Arcturus stream, an arch at $V \simeq-80 \mathrm{~km} \mathrm{~s}^{-1}$ is clearly visible inside a box defined by regions $01,11,21$, and 31 for the AF06 stream. In the $V-\sqrt{U^{2}+2 V^{2}}$ plane the structures are better resolved at scale $J=3$. In the angular momentum and action space there are strong detections of the high-velocity groups clearly visible at scale $J=3$. The Arcturus and KFR08 streams appear stronger at the smaller Galactocentric radii. Based on the analysis of all stars in action space, the Arcturus stream is an elongated structure in $L_{z}$. This means it covers a wider range of orbits, unlike the Hercules stream for example.

\section{The vertical extent of Arcturus and associated streams}

We focus here on three high-velocity structures (g1, g2, and g9) detected in region 00 in action space between $V \simeq-70$ and $-160 \mathrm{~km} \mathrm{~s}^{-1}$. The groups that we associate with these velocities in the solar region are the AF06, Arcturus, and KFR08 streams. We would like to know whether or not they are related, whether or not they are elongations of each other, and how different they are compared to the Hercules stream. The Hercules stream is chosen as a reference as it is one of the most studied kinematic structures and is a relatively metal-rich disc structure with the dynamical origin with the Galactic bar (e.g. Bensby et al. 2007; Ramya et al. 2016; Pérez-Villegas et al. 2017). One of the main peaks of the Hercules stream is group g18 detected in action space. We study properties of this group for a comparison with the high-velocity structures.

To further distinguish the three streams we investigate how the number density of stars in the Hercules, Arcturus, AF06, and KFR08 streams varies with vertical distance from the Galactic plane. Candidate member stars of the three streams were selected from the stellar sample constructed as described in Sect. 2 using the characteristic velocities of the streams that were found for region 00 (see Table A.2). From now on, it is assumed that kinematic groups are defined as stars on similar orbits. We assume that a star belongs to a group if its radial action and angular momentum fall into an ellipse around the centre of the group as shown in Fig. 3.

The leftmost plot of Fig. 7 shows the variation of the normalised number of stars in $\mathrm{g} 1, \mathrm{~g} 2$, g9, and g18 streams as defined in action space (see Fig. A.2) with the module of the distance from the Galactic plane $|Z|$ for region 00 . The Hercules stream is slightly more concentrated towards the Galactic plane compared to the three high-velocity structures. To check if this is valid in the regions outside the solar neighbourhood, stars in regions 01 , 11, 21, and 31 (see Fig. 2) that are members of groups g1, g2, $\mathrm{g} 9$, and $\mathrm{g} 18$ were selected. A star is defined as a member of a group if it has $J_{\mathrm{r}}$ and $L_{z}$ values located within an ellipse around a group in action space as shown in Fig. 2. Since actions are conserved quantities along orbits of stars in static potentials, it is expected that the structures will show up at the same positions in $L_{z}$ and $J_{\mathrm{r}}$. The right-hand side plot in Fig. 7 is the same as the one on the left, but for regions $01,11,21$, and 31. The Hercules stream is strongly concentrated to the Galactic plane and becomes rapidly weaker with distance from the plane. At distances above $|Z| \gtrsim 0.7 \mathrm{kpc}$, the density of stars in the Hercules stream drops to zero. In comparison, the g1, g2, and g9 structures reach larger heights from the plane. The disappearance of the Hercules stream after about $0.7 \mathrm{kpc}$ is consistent with the results from Antoja et al. (2012) that detected the Hercules stream at a lower confidence level at higher $Z$. To further probe the origins of the detected kinematic structures and how they relate to each other we make use of the detailed elemental abundance data from recent spectroscopic surveys.

\section{Chemical properties of the kinematic streams}

In this section we investigate whether or not the detected streams show distinct elemental abundance patterns. To our aim the detailed abundance data from large spectroscopic surveys such as GALAH DR2 (Buder et al. 2018) and APOGEE DR 14 (Holtzman et al. 2018) were used. GALAH DR2 includes over 340000 stars and APOGEE DR14 around 263000 stars. Both GALAH and APOGEE have determined radial velocities for all 

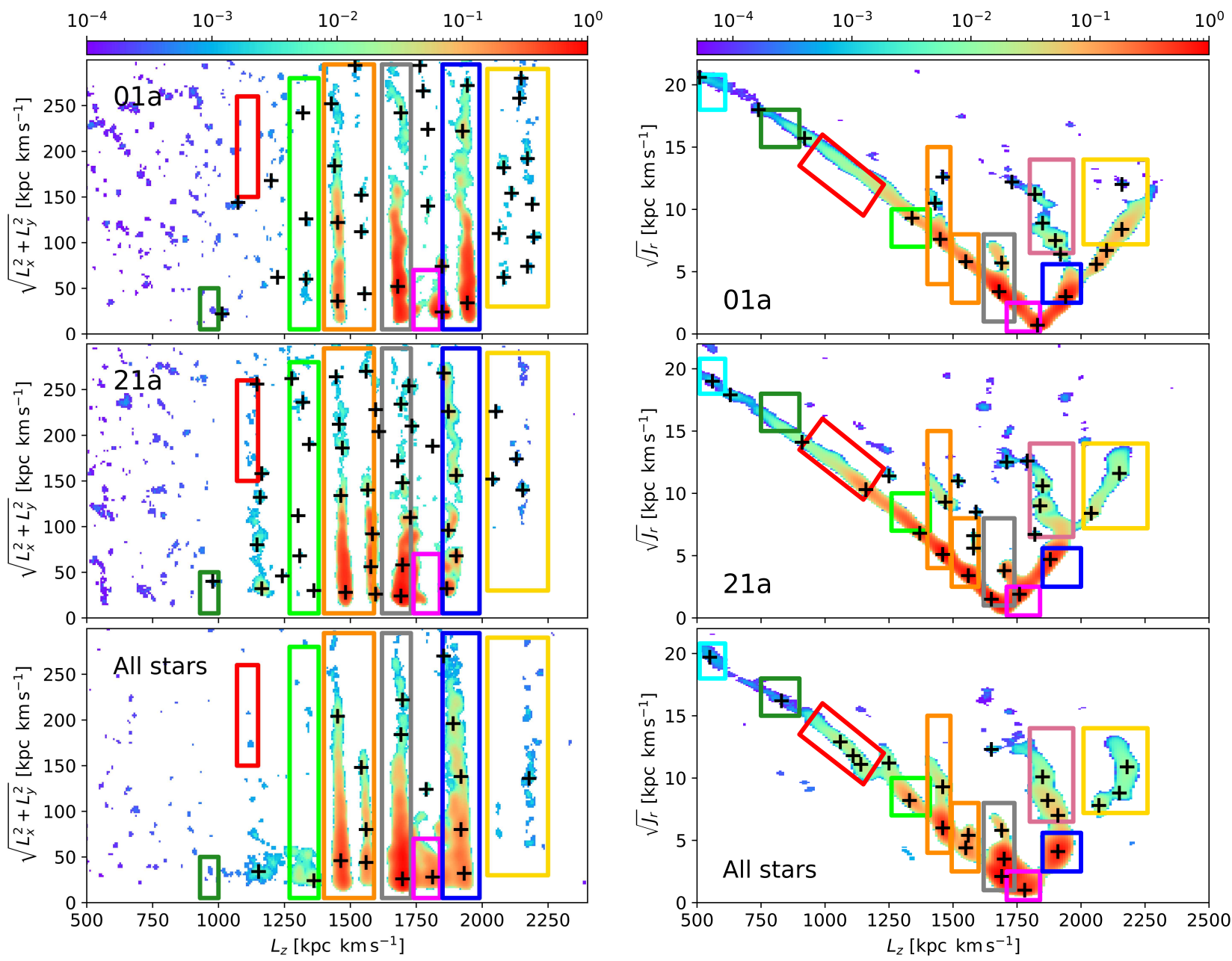

Fig. 6. Wavelet coefficient maps of regions 01a (top), 21a (middle) and all stars studied in this work (bottom) retrieved in $L_{z}-\sqrt{L_{x}^{2}+L_{y}^{2}}($ left column) and $L_{z}-\sqrt{J_{\mathrm{R}}}$ (right column) space for scale $J=2$. Colour bars show normalised wavelet coefficients. Black crosses show centres of the structures. Boxes around the crosses show location of kinematic structures based on the nearby sample 00 (see Fig. 3). Colours of the boxes correspond to different names of the structures listed in the legend of Fig. 3.

their targets, and those stars overlap to only a limited extent with the subsample of stars in Gaia that come with measured radial velocities in Gaia DR2. This means there will be just a few stars in each kinematic group when cross-matching GALAH and APOGEE with our sample that are constructed from Gaia DR2.

Therefore, to increase the number of stars that can be associated with the streams that were detected and that have elemental abundances in the GALAH and APOGEE data releases, we compute space velocities $U, V, W$, angular momenta $L_{x}, L_{y}, L_{z}$, and radial action $J_{\mathrm{r}}$ for all APOGEE and GALAH stars using astrometric data from Gaia DR2 and radial velocities from GALAH and APOGEE. The stars with $\sigma_{U}, \sigma_{V} \leq$ $20 \mathrm{~km} \mathrm{~s}^{-1}$ and with good quality flags were then selected. For GALAH, stars with good data quality flags were included: flag_cannon $=0$ and flag_x_fe $=0$, where $\mathrm{X}$ is a chemical element, and for APOGEE the following quality flags were used: $X \_F E \_F L A G=0$, where $X$ is a chemical element. This left us with a sample of 101862 and 72517 stars for the GALAH and APOGEE surveys, respectively. To select stars that are possible members of the detected kinematic streams, we use our kinematic constraints for action space listed in Table A.2, meaning that a star must be within a specific range in $L_{z}$ and $J_{\mathrm{r}}$ (i.e. must fall into an ellipse around the structure as shown in Fig. 2).
Figure 8 shows the $X-Y$ distributions for the constructed GALAH and APOGEE samples. It is seen that APOGEE covers more stars of the Northern sky and GALAH covers mainly the Southern part of the sky.

The top plots of Fig. 9 show the $[\alpha / \mathrm{Fe}]-[\mathrm{Fe} / \mathrm{H}]$ diagrams for stars in groups $\mathrm{g} 1, \mathrm{~g} 2, \mathrm{~g} 9$, and $\mathrm{g} 18$ selected in nine regions around the solar neighbourhood (01a_11a, 11a, 11a_21a, 01a, 00, 21a, 31a_01a, 31a, and 21a_31a) for GALAH and APOGEE samples. We over-plot results for these nine regions simply because there are not enough stars in the high-velocity streams in each region to present them separately. The stars members of the groups were selected based on the properties of the groups in region 00 listed in Table A.2. Since actions are conserved quantities, the groups are expected at the same positions after correcting $L_{z}$ values for the shift that arises due to differences in Galactocentric radii. The solid lines in these diagrams show the running mean for each stream. The shaded regions around each line show the corresponding $1 \sigma$ dispersions around the mean value.

The bottom plots of Fig. 9 show generalised metallicity distributions for the same groups as in the upper plots. Median values of the metallicity distributions and the corresponding dispersion of groups g18, g9, g2, and $\mathrm{g} 1$ are presented in Table 1 for the GALAH and APOGEE samples. The high-velocity streams generally have wide metallicity distributions and reaching lower metallicities 

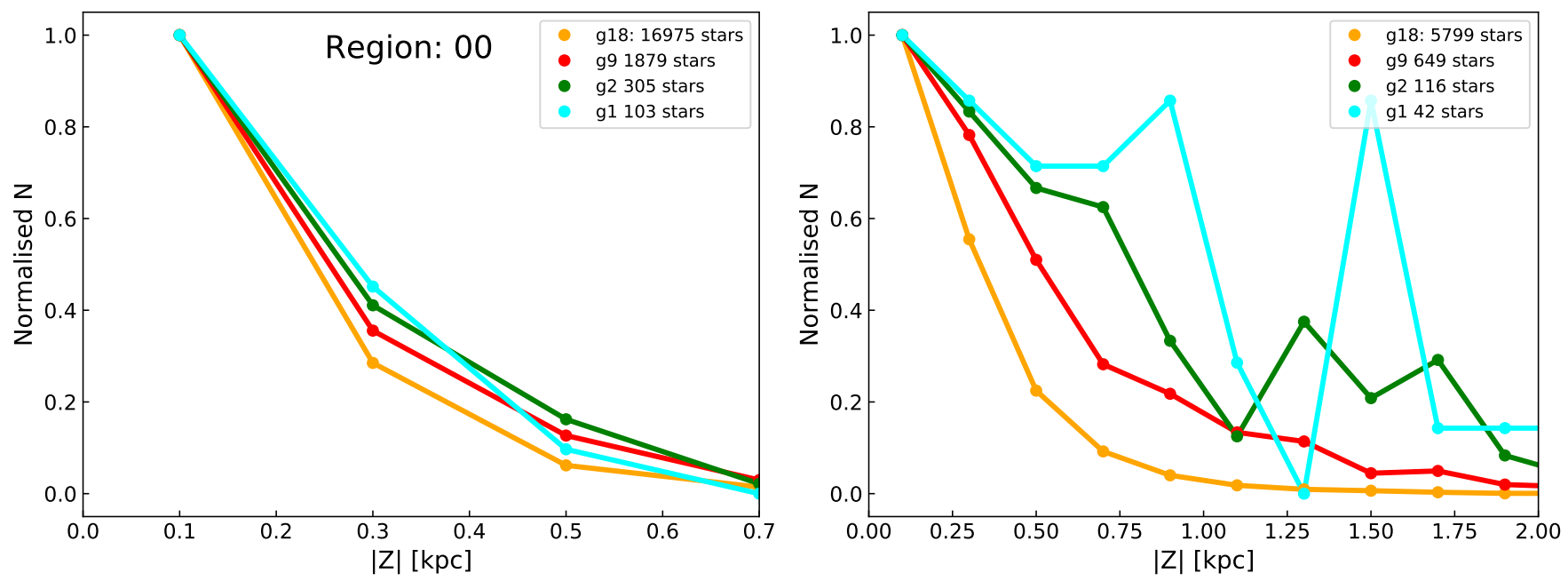

Fig. 7. Number of stars in Hercules, AF06, Arcturus, and KFR08 versus module of distance from the Galactic disc $|Z|$ in region 00 (left) and in regions $01,11,21$, and 31 (right).
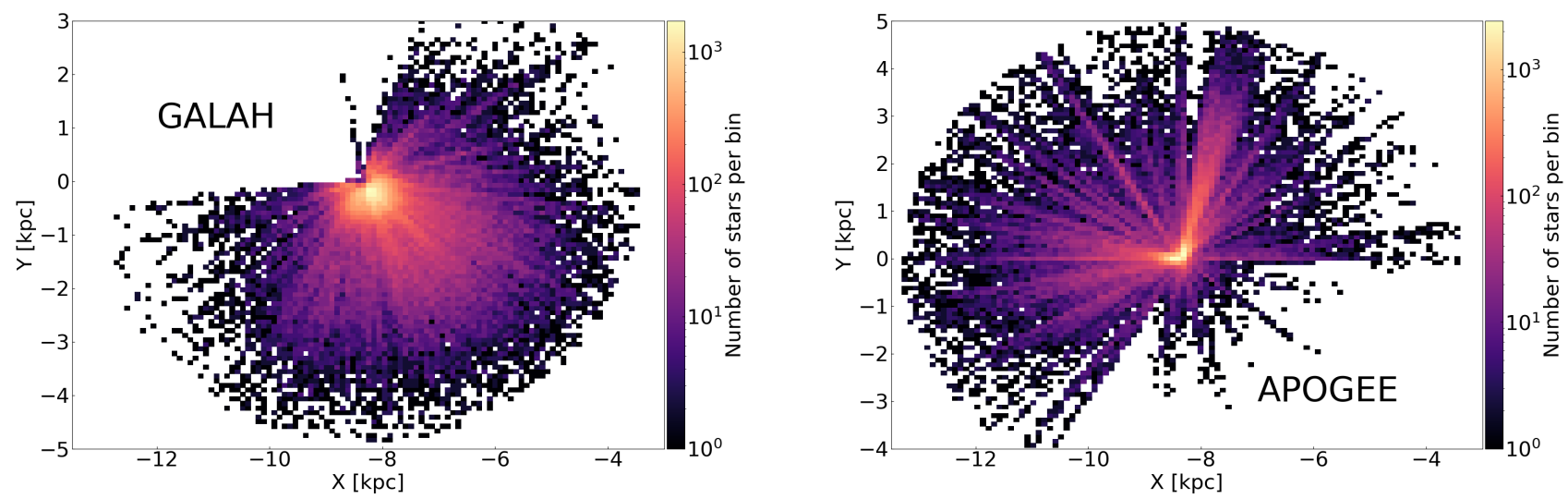

Fig. 8. $X-Y$ distributions for GALAH stars (left) and for APOGEE stars (right) with $\sigma_{U}, \sigma_{V} \leq 20 \mathrm{~km} \mathrm{~s}^{-1}$ and good quality flags. The bin size is $0.1 \mathrm{kpc}$ for both plots.

down to $[\mathrm{Fe} / \mathrm{H}] \lesssim-1$. The Hercules stream is more metal-rich. These plots show that the high-velocity streams could be highalpha thick-disc structures, while the Hercules stream is likely a mixture of both the thin and thick discs. Two-sided KolmogorovSmirnov tests were then used to check if the metallicity distribution of any of the streams come from the same distribution. In all cases the $p$-values were infinitesimally small, indicating that the null-hypothesis must be rejected, meaning that all the distributions are different. Results using APOGEE and GALAH surveys are similar: the high-velocity streams appear to be thick disc structures, while the Hercules stream is a mixture of both the thin and the thick-discs, and is a more metal-rich structure.

\section{The origin of the Arcturus stream}

In this section, we first provide a brief overview of the debates around the origin of the Arcturus stream, then summarise the kinematic and chemical characteristics of the Arcturus stream from this work, and based on that we discuss the possible origins of the stream.

\subsection{Accretion origin: debris of a disrupted satellite}

In Eggen $(1996,1998)$ it was shown that the Arcturus stream (then called a moving group) belongs to the old thick-disc population. It was fitted with a $10 \mathrm{Gyr}$ isochrone and the metallicity of Arcturus was estimated as $[\mathrm{Fe} / \mathrm{H}] \simeq-0.6$. Similar properties were observed by Gilmore et al. (2002) and Wyse et al. (2006) who found a clump of stars at $V \simeq-100 \mathrm{~km} \mathrm{~s}^{-1}$ that were estimated to be about 10-12 Gyr old and metal-poor with $-2.5<[\mathrm{Fe} / \mathrm{H}]<-0.5$. This is consistent with the properties of the Galactic thick disc.

One of the first attempts to explain the phenomenon of the Arcturus stream numerically was performed by Navarro et al. (2004). Assuming a merger event that happened 10-12 Gyr ago Navarro et al. (2004) obtained a structure with similar properties to the Arcturus stream. Navarro et al. (2004) also estimated the vertical component of the angular momentum of the group to be $L_{z} \simeq 1000 \mathrm{kpc} \mathrm{km} \mathrm{s}^{-1}$.

Further evidence for a possible debris origin for the Arcturus stream comes from Helmi et al. (2006), who found that a satellite galaxy with similar orbital properties to the Arcturus stream can produce three kinematic over-densities. One of the groups was linked to the Arcturus stream and investigated further through a detailed elemental abundance analysis by Ženovienè et al. (2014). These latter authors found that the average metallicity of the stream is $[\mathrm{Fe} / \mathrm{H}] \simeq-0.42$ and that its stars are about 8-12 Gyr old, which is consistent with the properties of the thick disc. Considering the results from Helmi et al. (2006), the Ženovienè et al. (2014) study supported a merger origin 

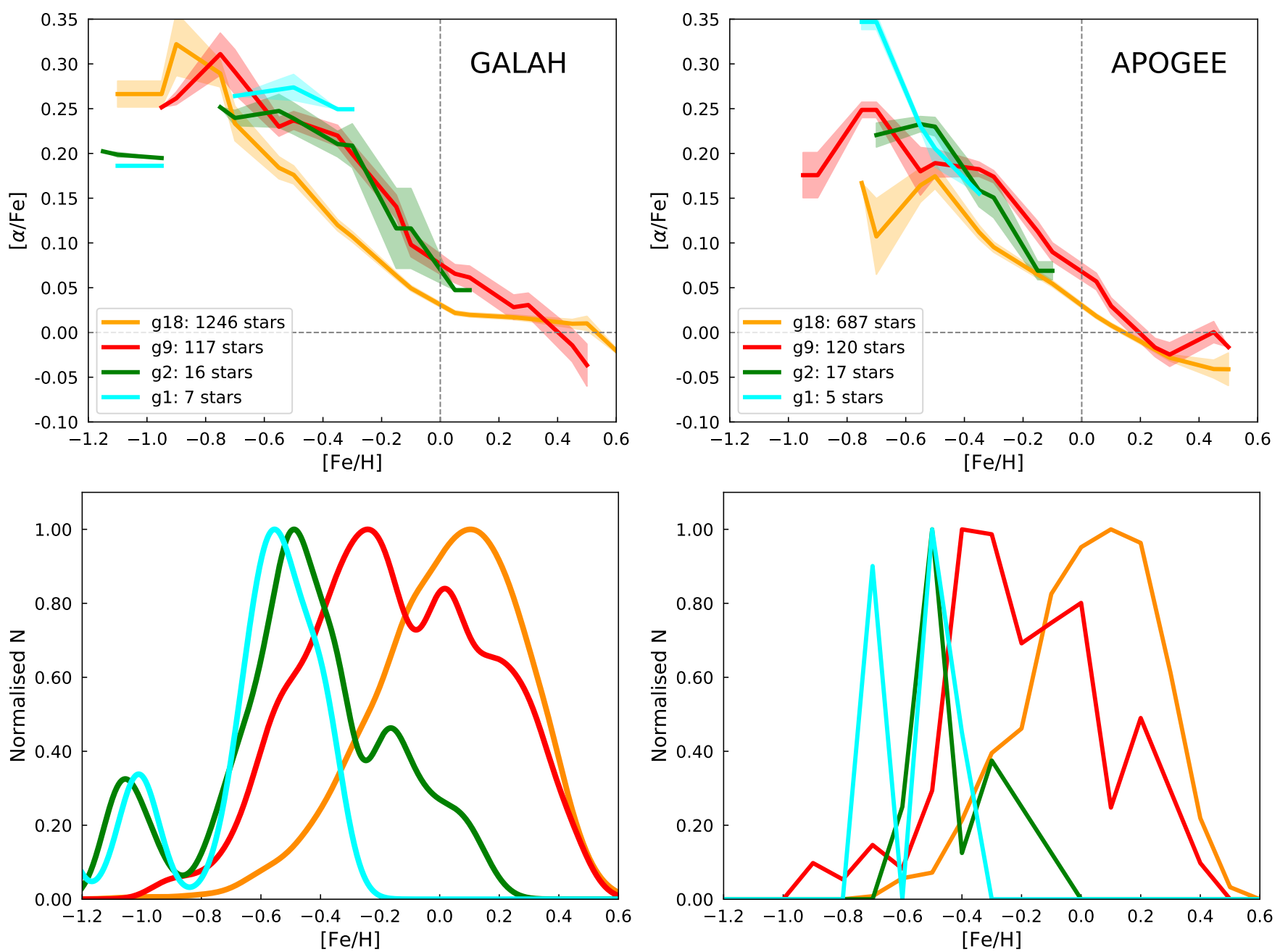

Fig. 9. Top plots: $[\mathrm{Fe} / \mathrm{H}]-[\alpha / \mathrm{Fe}]$ trends for the streams selected from the GALAH and APOGEE data in action space in a square of nine regions defined by regions 01a_11a, 11a_21a, 21a_31a and 31a_01a. The solid lines show the running means of the $[\alpha / \mathrm{Fe}]$ distributions in bins of [Fe/H], and the shaded regions give the 1- $\sigma$ dispersions of the data around the means. The bin width when calculating running means is 0.05 dex. Bottom plots: normalised generalised metallicity distributions for the stellar samples shown in the top plots. Here each star is represented by a Gaussian with a central peak at the estimated metallicity and a width given by the uncertainty of the metallicity.

Table 1. Median metallicities and corresponding dispersions of star members of the groups located in the square defined by nine regions around the central region 00 that were selected from APOGEE and GALAH samples.

\begin{tabular}{lrrrr}
\hline \hline \multirow{2}{*}{ Group } & \multicolumn{2}{c}{ GALAH } & \multicolumn{2}{c}{ APOGEE } \\
\cline { 2 - 5 } & {$[\mathrm{Fe} / \mathrm{H}]_{\text {median }}$} & $\sigma_{[\mathrm{Fe} / \mathrm{H}]}$ & {$[\mathrm{Fe} / \mathrm{H}]_{\text {median }}$} & $\sigma_{[\mathrm{Fe} / \mathrm{H}]}$ \\
\hline g18 & 0.0 & 0.2 & 0.0 & 0.2 \\
$\mathrm{~g} 9$ & -0.2 & 0.3 & -0.2 & 0.3 \\
$\mathrm{~g} 2$ & -0.5 & 0.3 & -0.5 & 0.2 \\
$\mathrm{~g} 1$ & -0.6 & 0.3 & -0.5 & 0.1 \\
\hline
\end{tabular}

for the stream. On the other hand, a comparison with another detailed elemental abundance study by Ramya et al. (2012), who applied different selection criteria for possible Arcturus stream stars, suggested that it is different from the thick disc and the two groups studied in these works are different.

An alternative approach to search for kinematic overdensities was proposed by Arifyanto \& Fuchs (2006), and then followed by Klement et al. (2008) and Zhao et al. (2014). Using wavelet transforms they searched for clumps in the plane defined by the $\sqrt{U^{2}+2 V^{2}}$ and $V$ space velocities, where $U$ and $V$ are radial and tangential velocity components, respectively. These latter authors detected many kinematic structures in the range $-200 \leq V \leq-80 \mathrm{~km} \mathrm{~s}^{-1}$, including candidates for the Arcturus stream. Following the approach proposed by Helmi et al. (1999), Klement et al. (2008), and Zhao et al. (2014) studied angular momenta space defined by $\sqrt{L_{x}^{2}+L_{y}^{2}}$ and $L_{z}$, the angular momenta components of the stars. They placed the Arcturus stream at $V \simeq-100 \mathrm{~km} \mathrm{~s}^{-1}$ and $L_{z} \simeq 1000 \mathrm{kpc} \mathrm{km} \mathrm{s}^{-1}$. The high eccentricity and low metallicity of the high-velocity streams lead to a conclusion that the Arcturus has a merger debris origin.

The above papers provide evidence that the Arcturus stream and other high-velocity streams can be explained as debris from disrupted satellite galaxies that merged with the Milky Way in the past.

\subsection{Galactic origin: resonances}

If the debris origin for the observed streams is correct, the stars within a stream should have a distinct elemental abundance pattern different from what is observed for the disc stars of the 
Milky Way. A detailed chemical analysis of possible members of the Arcturus stream and another group called the AF06 stream, first assigned by Arifyanto \& Fuchs (2006), was performed by Ramya et al. (2012). No unique chemical features were found for either of the groups; their chemical compositions are similar to the background thick disc stars, being metal-poor, alphaenriched, and have ages between 10 and 14 Gyr. This indicates that these structures are likely to have a dynamical origin within the Galaxy.

Another chemical analysis of the Arcturus stream was performed by Williams et al. (2009). The stellar sample was constructed based on results of the N-body simulations, where a satellite was accreted by the Milky Way. It was also found by these latter authors that the stream stars are chemically inhomogeneous, being similar to the thick disc, and thus, cannot be called a moving group. The authors discuss a possible origin of the group within the Milky Way, being dynamically formed due to Lindblad resonances. At the same time they do not reject the possibility of a merger origin.

Bensby et al. (2014) studied ages and chemical composition of the Galactic disc stars and briefly explored those that could potentially belong to the Arcturus stream $(-115<V<$ $-85 \mathrm{~km} \mathrm{~s}^{-1}$ ). These latter authors found no chemical signature of a merger event, but rather that a dynamical origin is more probable due to similarities of chemical patterns of the group with the thick disc.

If the discussed spectroscopic studies question an accretion origin for the Arcturus, can the structure be reproduced in a resonant scenario via numerical simulations? Assuming resonances with the Galactic long bar, Gardner \& Flynn (2010) simulated a kinematic group which has properties similar to the Arcturus stream. Numerical simulations performed by Monari et al. (2013) show a similar result; the Galactic long bar can produce a feature that is consistent with the Arcturus stream.

All these findings lead to the question of whether the Arcturus stream formed due to resonances or due to a merger event. Simulations assuming either hypothesis are able to reproduce a phase-space structure similar to the Arcturus stream. At the same time there is no clear consensus from the detailed elemental abundance studies.

\subsection{Other hypothesis and recent findings}

An alternative opinion on the origin of the Arcturus stream was proposed by Minchev et al. (2009). Assuming the existence of a dynamically unrelaxed population which formed after a merger event, these latter authors simulated how the distribution of stars in the $U-V$ and $V-\sqrt{U^{2}+2 V^{2}}$ changes with time. They found ring-like structures that represent a wave with streams appearing almost every $20 \mathrm{~km} \mathrm{~s}^{-1}$ in $V$, placing the Arcturus stream at $-100 \mathrm{~km} \mathrm{~s}^{-1}$. Based on the kinematics of the simulated structures, the authors state that the Galactic disc was perturbed about 1.9 Gyr ago and match it with the time when the Galactic bar could have formed.

Another support of the ringing hypothesis came with the Gaia DR2 (Gaia Collaboration 2018a) release. The analysis of Gaia DR2 data revealed a rich arch- and ridge-like substructure in the phase-space that is strong evidence that the disc of the Milky Way is far from being in equilibrium and undergoes phase-mixing (Antoja et al. 2018; Ramos et al. 2018; Monari et al. 2018; Tian et al. 2018). This phase-mixing could be a result of external-perturbations due to a passage of the Sagittarius dwarf galaxy (e.g. Antoja et al. 2018). Indeed, Laporte et al. (2019) demonstrated, using a state-of-the-art simulation of the Milky Way-Sagittarius interaction, that a range of complex phase-space structures discovered in Gaia DR2 data can be explained simultaneously by their model existing prior to the Gaia DR2 data release, including the disk in-plane velocity field (Katz et al. 2019), the ridges in the $V_{\phi}-R$ plane (Antoja et al. 2018; Kawata et al. 2018), and the spiral in the $Z-V_{z}$ plane (Antoja et al. 2018). Here, $V_{\phi}$ and $V_{z}$ are velocities in the Galactocentric coordinate system. At the same time, Hunt et al. (2018), Quillen et al. (2018), and Sellwood et al. (2019), show that the phase-space ridges could be a result of an impact of the Galactic spiral arms, but the simulations do not cover the high-velocity field. The Arcturus stream is one of the arch-like structures seen in Ramos et al. (2018), who performed a deeper study of the substructures with the wavelet analysis. If the Arcturus is a kinematic wave in the Galactic disc, then what triggers the formation of these structures, the bar, spiral arms or a merger event? What is the nature of the Arcturus stream and how similar is it to the other kinematic structures?

\subsection{The origin of the Arcturus stream in this study}

Before discussing possibilities for the nature and origin of the Arcturus stream (g2), we summarise the properties of the kinematic substructures associated with the Arcturus stream that we have found so far:

- The rotational velocity of the Arcturus stream is $V \simeq$ $-127 \mathrm{~km} \mathrm{~s}^{-1}$, and the vertical component of the angular momentum is $L_{z} \simeq 840 \mathrm{kpc} \mathrm{km} \mathrm{s}^{-1}$. This is in agreement with the results from Navarro et al. (2004) who found the Arcturus stream at $V \simeq-100 \mathrm{~km} \mathrm{~s}^{-1}$ and $L_{z} \simeq$ in the range between 700 and $1100 \mathrm{kpckm} \mathrm{s}^{-1}$, Klement et al. (2008) who found it at $V \simeq$ $-120 \mathrm{~km} \mathrm{~s}^{-1}$ and $L_{z} \simeq 1000 \mathrm{kpc} \mathrm{km} \mathrm{s}^{-1}$, and Ramya et al. (2012) who found it at $V \simeq-125 \mathrm{~km} \mathrm{~s}^{-1}$ and $L_{z} \simeq 811 \mathrm{kpc} \mathrm{km} \mathrm{s}^{-1}$.

- The Arcturus stream (g2), as well as the AF06 and KFR08 streams (g1 and g9), are not only detected in the solar neighbourhood. The structures appear in a larger box defined by regions 01_12, 22_12, 22_32, and 02_32. The remaining regions contain significantly less stars and their distance uncertainties are larger (see Table A.1). Considering the fact stated above it is not possible to definitely answer whether or not there are kinematic structures in those regions, or if they are weaker, or if it is a consequence of the properties of the stellar sample.

- The Arcturus stream extends to about $2 \mathrm{kpc}$ vertically from the Galactic plane. At the same time the stars of the Hercules stream appear to be disappearing at distances greater than $|Z| \gtrsim 1 \mathrm{kpc}$. The streams detected at even higher $V$ velocities extend to even greater distances from the Galactic plane. This is consistent with the results from Antoja et al. (2012) who found that the Hercules stream has a lower detection level after $|Z| \gtrsim 0.6 \mathrm{kpc}$.

- The Arcturus, KFR08, and AF06 streams are alphaenhanced in the $[\alpha / \mathrm{Fe}]-[\mathrm{Fe} / \mathrm{H}]$ diagram, and show similarities to the Galactic thick disc. They are clearly different from the Hercules stream which appears to be a mixture of the thin and thick discs. This is in agreement with, for example Bensby et al. (2007, 2014), Williams et al. (2009), and Ramya et al. (2012).

- The Arcturus stream has a wide metallicity distribution spanning the interval $-1.2 \leq[\mathrm{Fe} / \mathrm{H}] \leq 0.2$ and peaking at $\simeq-0.5$, which is not different from what has been found in other studies. Eggen (1996) found $[\mathrm{Fe} / \mathrm{H}] \simeq-0.6$, and Ramya et al. (2012) found a more metal-poor range for the Arcturus stream, $-1.4 \leq[\mathrm{Fe} / \mathrm{H}] \leq-0.37$, peaking at $[\mathrm{Fe} / \mathrm{H}] \simeq-0.7$. 
Considering the chemical properties of the Arcturus stream (g2) we see that it is not a chemically homogeneous structure. This indicates that the Arcturus stream is not a moving group, similarly to what was found by Williams et al. (2009), Ramya et al. (2012), and Bensby et al. (2014). Also, the metallicity distributions for the Arcturus, KFR08, and AF06 streams (g2, g1, and $\mathrm{g} 9$, respectively) are too wide for them to be called moving groups. At the same time, the mean metallicities are different for each stream, meaning that they appear to be independent structures, which is different from the conclusion by Zhao et al (2014) that the Arcturus and AF06 streams should be regarded as one structure. It was also shown that a two-sided K-S tests rejected the hypothesis that any of the groups could come from the same population.

Groups g1, g2, and g9 found in this work appear, at least chemically, to be thick disc structures. Therefore, we question the debris origin for the streams. If the streams have a debris origin, their chemical compositions should be different from the thick disc stars. This is different from the Liu et al. (2015) conclusion of a merger origin for the KFR08 stream. It is clearly seen that KFR08 has a wide metallicity distribution and its kinematics and chemistry are consistent with what is seen for the stars in the Galactic thick disc.

The two remaining possibilities for the origin of the Arcturus stream and the neighbouring KFR08 and AF06 streams (g2, g1 and $\mathrm{g} 9$ respectively) are the external-perturbation origin or being due to the resonances with the spiral arms or the Galactic bar. The results we have present here are consistent with the results from Minchev et al. (2009), who simulated the velocity distribution in the $V-\sqrt{U^{2}+2 V^{2}}$ and $U-V$ planes assuming a merger event that perturbed the Galactic disc and caused an ongoing phase-mixing, inducing kinematic over-densities to be placed on the $V$ axis every $20 \mathrm{~km} \mathrm{~s}^{-1}$. This is essentially what we observe in this work. Arch-like structures are easily recognisable in Fig. 3 and are similar to what was found in for example Gaia Collaboration (2018b), Antoja et al. (2018), and Ramos et al. (2018). The patterns discussed in Minchev et al. (2009) are observed at the $V-\sqrt{U^{2}+2 V^{2}}$ and action space; the arches observed in the $U-V$ plane become clumps. These clumps and arches are observed as lines in the angular momentum space. Within the uncertainties these features (arches, clumps, and lines) show up 20-30 $\mathrm{km} \mathrm{s}^{-1}$ apart in the $V$ velocity component. According to Minchev et al. (2009), this could be due to ongoing mixing in the disc after a merger event. The fact that the highvelocity groups extend to higher $|Z|$ than the bar-originated structures leads us to conclude on an external-perturbation (phasemixing) origin for the Arcturus stream and its neighbour KFR08 and AF06 streams is a possible scenario.

\section{Summary}

In order to resolve the nature of the Arcturus stream we analysed the velocity and angular momenta distributions of the Gaia DR2 stars at different Galactocentric radii. The analysis revealed the following:

- The analysis of four spaces defined by velocity, angular momentum, and action components in 65 smaller volumes allowed us to detect the previously well-studied Sirius, Pleiades, Hyades, and Hercules streams; a few high-velocity structures that are associated with the AF06, Arcturus, and KFR08 streams; and many unknown clumps that might be a part of larger streams.

- The picture observed in the velocity space is consistent with the results from Minchev et al. (2009). Their model predicts kinematic structures to be placed every $20-30 \mathrm{~km} \mathrm{~s}^{-1}$ in $V$. This is similar to what was observed with the Gaia DR2 sample: starting with the Sirius at $0 \mathrm{~km} \mathrm{~s}^{-1}$ and ending with the KFR08 stream at $\simeq-160 \mathrm{~km} \mathrm{~s}^{-1}$ we observe kinematic structures every $V \simeq 20-30 \mathrm{~km} \mathrm{~s}^{-1}$ (taking into account velocity uncertainties and the sizes of the structures).

- The arches observed in the $U-V$ plane are observed as clumps in the $V-\sqrt{U^{2}+2 V^{2}}$ and action space and lines in the angular momentum space. Minchev et al. (2009) as well as Antoja et al. (2018) link these arches to the ongoing phasemixing in the disc of the Milky Way due to a strong disc perturbation, likely originating from a merger event.

- The high-velocity streams are observed at higher $|Z|$ compared to the Hercules stream which is currently considered to be a structure caused by the Galactic bar (e.g. Antoja et al. 2014; Pérez-Villegas et al. 2017). The KFR08 stream, which has the highest values of $V$ and lowest $L_{z}$ extends at least $2 \mathrm{kpc}$ further from the Galactic disc, while the Hercules is located closer to the Galactic disc $|Z|<0.7 \mathrm{kpc}$.

- The majority of stars from the sample analysed in this work are within $1 \mathrm{kpc}$ in $|Z|$ and according to Monari et al. (2013) the Galactic bar can influence stellar motion up to $|Z| \simeq 1 \mathrm{kpc}$ in the thin disc and up to $|Z| \simeq 2 \mathrm{kpc}$. However, the bar-induced arches do not cover the observed range of about $\pm 100 \mathrm{~km} \mathrm{~s}^{-1}$ in radial Galactocentric velocity $V_{\mathrm{r}}$.

- The high-velocity groups are present mainly in the nearby regions. This is consistent with Ramos et al. (2018), where one of the discovered arches, that the authors associate with the Arcturus group, is located mainly in the solar neighbourhood and within the solar circle. At the same time this result could be the consequence of the fact that nearby regions contain more stars and distance uncertainties are smaller.

- Ramos et al. (2018) discuss the negative gradients of the rotational velocity of the structures with the Galactocentric radii. This gradient should be positive for the Cartesian velocities $V$. We do observe similar gradients in the $U-V$ plane.

- The analysis of the chemical abundances of stars that are members of the groups taken from the APOGEE and GALAH spectroscopic surveys confirmed that the AF06, Arcturus, and KFR08 groups have chemical patterns that resemble that of the thick disc. The groups cover wider metallicity ranges compared to Hercules, which appears to be a mixture of the thin and thick disc stars, which is in agreement with the literature (e.g. Bensby et al. 2014; Ramya et al. 2016). The estimated median metallicity of Hercules in this work is $[\mathrm{Fe} / \mathrm{H}] \simeq 0.0$. In comparison, Ramya et al. (2016), for example, obtained $[\mathrm{Fe} / \mathrm{H}] \simeq 0.15$.

- The two-sided K-S test performed for different combinations of the groups (Hercules, AF06, Arcturus, KFR08) rejected the possibility for all of them to be drawn from the same distribution.

Arcturus, KFR08, and AF06 are kinematic structures that have rotational $V$ velocities separated with a fixed step, extend farther from the Galactic plane compared to other over-densities such as the Hercules stream, and have chemical compositions consistent with the properties of the Galactic thick disc. Collectively, this points towards an origin for the structures related to the ongoing kinematic mixing or ringing in the disc, as was suggested in Minchev et al. (2009). The recently discovered ridges and arches in the phase-space (Antoja et al. 2018; Ramos et al. 2018) found with the Gaia DR2, including the Arcturus arch, represent further evidence that the high-velocity kinematic structures such as the Arcturus stream could be a result of the external-perturbation process and were formed inside the Milky Way. 
Though a great amount of effort has been made to understand the nature of these phase-space waves with the Gaia DR2 data, we are still far from an unambiguous answer. Do these waves have a merger origin as was originally proposed by Minchev et al. (2009), or are they caused by the spiral arms as suggested, for example, by Quillen et al. (2018), Hunt et al. (2018), and Sellwood et al. (2019)? Numerical simulations together with the chemical abundances from spectroscopic surveys like the Gaia-ESO survey (Gilmore et al. 2012), WEAVE (Dalton et al. 2014), and 4MOST (de Jong et al. 2019), in combination with upcoming Gaia data releases, will broaden the opportunities for us to better understand the formation of the phase-space warps and might provide a definitive answer about the origin of kinematic structures like the Arcturus stream. Current observational evidence however points towards a phase-space mixing origin.

Acknowledgements. We thank Prof. F. Murtagh for making available for us the MR software packages and Dr. P. J. McMillan for a valuable help when needed T. B. was funded by the project grant "The New Milky Way" from the Knut and Alice Wallenberg foundation.

\section{References}

Antoja, T., Figueras, F., Fernández, D., \& Torra, J. 2008, A\&A, 490, 135 Antoja, T., Helmi, A., Bienayme, O., et al. 2012, MNRAS, 426, L1 Antoja, T., Helmi, A., Dehnen, W., et al. 2014, A\&A, 563, A60 Antoja, T., Helmi, A., Romero-Gómez, M., et al. 2018, Nature, 561, 360 Arenou, F., Luri, X., Babusiaux, C., et al. 2018, A\&A, 616, A17 Arifyanto, M. I., \& Fuchs, B. 2006, A\&A, 449, 533

Bailer-Jones, C. A. L. 2015, PASP, 127, 994

Bensby, T., Oey, M. S., Feltzing, S., \& Gustafsson, B. 2007, ApJ, 655, L89

Bensby, T., Feltzing, S., \& Oey, M. S. 2014, A\&A, 562, A71

Bobylev, V. V., \& Bajkova, A. T. 2016, Astron. Lett., 42, 90

Bovy, J. 2015, ApJS, 216, 29

Bovy, J. 2016, ApJ, 817, 49

Buder, S., Asplund, M., Duong, L., et al. 2018, MNRAS, 478, 4513

Chakrabarty, D. 2007, A\&A, 467, 145

Dalton, G., Trager, S., Abrams, D. C., et al. 2014, Ser., 9147, 91470L de Jong, R. S., Agertz, O., Berbel, A. A., et al. 2019, The Messenger, 175, 3 De Silva, G. M., Freeman, K. C., Bland-Hawthorn, J., Asplund, M., \& Bessell, M. S. 2007, AJ, 133, 694

Dehnen, W. 1998, AJ, 115, 2384

Dehnen, W. 2000, AJ, 119, 800

Dekker, E. 1976, Phys. Rep., 24, 315

Eggen, O. J. 1971, PASP, 83, 762

Eggen, O. J. 1996, AJ, 112, 1595

Eggen, O. J. 1998, AJ, 115, 2397

Famaey, B., Jorissen, A., Luri, X., et al. 2005, A\&A, 430, 165

Feltzing, S., \& Holmberg, J. 2000, A\&A, 357, 153

Gaia Collaboration (Brown, A. G. A., et al.) 2018a, A\&A, 616, A1

Gaia Collaboration (Katz, D., et al.) 2018b, A\&A, 616, A11

Gardner, E., \& Flynn, C. 2010, MNRAS, 405, 545

Gilmore, G., Wyse, R. F. G., \& Norris, J. E. 2002, ApJ, 574, L39
Gilmore, G., Randich, S., Asplund, M., et al. 2012, The Messenger, 147, 25

Helmi, A., White, S. D. M., de Zeeuw, P. T., \& Zhao, H. 1999, Nature, 402, 53 Helmi, A., Navarro, J. F., Nordström, B., et al. 2006, MNRAS, 365, 1309

Helmi, A., Veljanoski, J., Breddels, M. A., Tian, H., \& Sales, L. V. 2017, A\&A, 598, A58

Helmi, A., Babusiaux, C., Koppelman, H. H., et al. 2018, Nature, 563, 85

Holtzman, J. A., Hasselquist, S., Shetrone, M., et al. 2018, AJ, 156, 125

Hunt, J. A. S., Hong, J., Bovy, J., Kawata, D., \& Grand, R. J. J. 2018, MNRAS, 481, 3794

Johnson, D. R. H., \& Soderblom, D. R. 1987, AJ, 93, 864

Katz, D., Sartoretti, P., Cropper, M., et al. 2019, A\&A, 622, A205

Kawata, D., Baba, J., Ciucă, I., et al. 2018, MNRAS, 479, L108

Klement, R., Fuchs, B., \& Rix, H.-W. 2008, ApJ, 685, 261

Koppelman, H., Helmi, A., \& Veljanoski, J. 2018, ApJ, 860, L11

Kushniruk, I., Schirmer, T., \& Bensby, T. 2017, A\&A, 608, A73

Laporte, C. F. P., Minchev, I., Johnston, K. V., \& Gómez, F. A. 2019, MNRAS, 485,3134

Lindegren, L. 2018, Gaia Technical Note: GAIA-C3-TN-LU-LL-124-01

Liu, C., Feltzing, S., \& Ruchti, G. 2015, A\&A, 580, A111

McMillan, P. J. 2018, Res. Notes Am. Astron. Soc., 2, 51

Minchev, I., Nordhaus, J., \& Quillen, A. C. 2007, ApJ, 664, L31

Minchev, I., Quillen, A. C., Williams, M., et al. 2009, MNRAS, 396, L56

Minchev, I., Boily, C., Siebert, A., \& Bienayme, O. 2010, MNRAS, 407, 2122

Monari, G., Antoja, T., \& Helmi, A. 2013, ArXiv e-prints [arXiv:1306.2632]

Monari, G., Kawata, D., Hunt, J. A. S., \& Famaey, B. 2017, MNRAS, 466, L113

Monari, G., Famaey, B., Minchev, I., et al. 2018, Res. Notes Am. Astron. Soc., 2,32

Monari, G., Famaey, B., Siebert, A., et al. 2019, in press, https://doi .org/ 10.1051/0004-6361/201936455

Navarro, J. F., Helmi, A., \& Freeman, K. C. 2004, ApJ, 601, L43

Pérez-Villegas, A., Portail, M., Wegg, C., \& Gerhard, O. 2017, ApJ, 840, L2

Quillen, A. C., \& Minchev, I. 2005, AJ, 130, 576

Quillen, A. C., Carrillo, I., Anders, F., et al. 2018, MNRAS, 480, 3132

Ramos, P., Antoja, T., \& Figueras, F. 2018, A\&A, 619, A72

Ramya, P., Reddy, B. E., \& Lambert, D. L. 2012, MNRAS, 425, 3188

Ramya, P., Reddy, B. E., Lambert, D. L., \& Musthafa, M. M. 2016, MNRAS, 460, 1356

Schönrich, R., Binney, J., \& Dehnen, W. 2010, MNRAS, 403, 1829

Sellwood, J. A. 2010, MNRAS, 409, 145

Sellwood, J. A., Trick, W. H., Carlberg, R. G., Coronado, J., \& Rix, H.-W. 2019, MNRAS, 484, 3154

Skuljan, J., Hearnshaw, J. B., \& Cottrell, P. L. 1999, MNRAS, 308, 731

Starck, J.-L., \& Murtagh, F. 2002, Astronomical Image and Data Analysis (Berlin: Springer)

Starck, J. L., \& Murtagh, F. D. 1998, Image Processing and Data Analysis (Cambridge: Cambridge University Press), 297

Tian, H.-J., Liu, C., Wu, Y., Xiang, M.-S., \& Zhang, Y. 2018, ApJ, 865, L19

Trick, W. H., Coronado, J., \& Rix, H.-W. 2019, MNRAS, 484, 3291

van der Walt, S., Schönberger, J. L., Nunez-Iglesias, J., et al. 2014, PeerJ, 2, e453

Wegg, C., Gerhard, O., \& Portail, M. 2015, MNRAS, 450, 4050

Williams, M. E. K., Freeman, K. C., Helmi, A., \& RAVE Collaboration 2009, in The Galaxy Disk in Cosmological Context, eds. J. Andersen, B. M. Nordströara, \& J. Bland-Hawthorn, IAU Symp., 254, 139

Wyse, R. F. G., Gilmore, G., Norris, J. E., et al. 2006, ApJ, 639, L13

Ženovienè, R., Tautvaišienè, G., Nordström, B., \& Stonkutè, E. 2014, A\&A, 563, A53

Zhao, J. K., Zhao, G., Chen, Y. Q., et al. 2014, ApJ, 787, 31 
I. Kushniruk and T. Bensby: Disentangling the Arcturus stream

\section{Appendix A: Additional tables}

Table A.1. Number of stars, median distance, and median distance uncertainty for the stars located in the 65 regions (as defined in Fig. 1).

\begin{tabular}{|c|c|c|c|c|}
\hline$N$ & Region & $N$ stars & $\begin{array}{r}D_{\text {median }} \\
{[\mathrm{pc}]}\end{array}$ & $\begin{array}{r}\sigma_{D_{\text {median }}} \\
{[\mathrm{pc}]}\end{array}$ \\
\hline 1 & 00 & 666505 & 212 & 2 \\
\hline 2 & 01 & 48759 & 851 & 45 \\
\hline 3 & 02 & 13647 & 1689 & 181 \\
\hline 4 & 03 & 6332 & 2483 & 386 \\
\hline 5 & 04 & 3041 & 3285 & 677 \\
\hline 6 & 05 & 1621 & 4083 & 932 \\
\hline 7 & 11 & 70747 & 861 & 38 \\
\hline 8 & 12 & 22070 & 1778 & 164 \\
\hline 9 & 13 & 8991 & 2636 & 375 \\
\hline 10 & 14 & 3521 & 3502 & 669 \\
\hline 11 & 15 & 1685 & 4338 & 918 \\
\hline 12 & 21 & 81838 & 777 & 38 \\
\hline 13 & 22 & 24823 & 1602 & 171 \\
\hline 14 & 23 & 10146 & 2392 & 410 \\
\hline 15 & 24 & 3307 & 3204 & 803 \\
\hline 16 & 25 & 1386 & 4011 & 1171 \\
\hline 17 & 31 & 64875 & 868 & 38 \\
\hline 18 & 32 & 19806 & 1778 & 164 \\
\hline 19 & 33 & 10747 & 2624 & 365 \\
\hline 20 & 34 & 4307 & 3489 & 655 \\
\hline 21 & 35 & 1873 & 4329 & 826 \\
\hline 22 & 22_11 & 18596 & 1798 & 209 \\
\hline 23 & 22_12 & 13098 & 2258 & 306 \\
\hline 24 & 22_13 & 8223 & 2853 & 495 \\
\hline 25 & 22_14 & 4132 & 3522 & 724 \\
\hline 26 & 22_15 & 2197 & 4216 & 898 \\
\hline 27 & 22_31 & 27838 & 1780 & 194 \\
\hline 28 & 22_32 & 18135 & 2242 & 284 \\
\hline 29 & 22_33 & 9545 & 2839 & 458 \\
\hline 30 & 22_34 & 5646 & 3507 & 690 \\
\hline 31 & $22 \_35$ & 2832 & 4200 & 922 \\
\hline 32 & 02_11 & 11447 & 1942 & 215 \\
\hline 33 & 02_12 & 8189 & 2546 & 349 \\
\hline 34 & 02_13 & 3219 & 3321 & 608 \\
\hline 35 & 02_14 & 2267 & 4145 & 852 \\
\hline 36 & 02_15 & 308 & 4897 & 954 \\
\hline 37 & 02_31 & 12179 & 1939 & 232 \\
\hline 38 & 02_32 & 9594 & 2544 & 372 \\
\hline 39 & 02_33 & 4786 & 3306 & 602 \\
\hline 40 & 02_34 & 1887 & 4147 & 868 \\
\hline 41 & 02_35 & 263 & 4881 & 903 \\
\hline 42 & 12_01 & 13658 & 2042 & 217 \\
\hline 43 & 12_03 & 4287 & 3177 & 589 \\
\hline 44 & 12_04 & 1942 & 3893 & 847 \\
\hline 45 & 12_05 & 1125 & 4605 & 1024 \\
\hline 46 & 12_21 & 21401 & 1864 & 189 \\
\hline 47 & 12_23 & 6129 & 2823 & 538 \\
\hline 48 & 12_24 & 2440 & 3500 & 896 \\
\hline 49 & 12_25 & 1195 & 4206 & 1173 \\
\hline
\end{tabular}

Table A.1. continued.

\begin{tabular}{|c|c|c|c|c|}
\hline$N$ & Region & $N$ stars & $\begin{array}{r}D_{\text {median }} \\
{[\mathrm{pc}]}\end{array}$ & $\begin{array}{r}\sigma_{D_{\text {median }}}[\mathrm{pc}] \\
\end{array}$ \\
\hline 50 & 32_01 & 13717 & 2044 & 219 \\
\hline 51 & 32_03 & 3988 & 3176 & 609 \\
\hline 52 & 32_04 & 2172 & 3871 & 829 \\
\hline 53 & 32_05 & 1290 & 4592 & 1081 \\
\hline 54 & 32_21 & 22441 & 1856 & 186 \\
\hline 55 & 32_23 & 3331 & 3496 & 855 \\
\hline 56 & 32_24 & 11387 & 2806 & 495 \\
\hline 57 & 32_25 & 1447 & 4209 & 1166 \\
\hline 58 & $\overline{0} 1 \mathrm{a}$ & 211846 & 453 & 12 \\
\hline 59 & $11 \mathrm{a}$ & 271059 & 444 & 10 \\
\hline 60 & $21 \mathrm{a}$ & 317397 & 387 & 9 \\
\hline 61 & $31 a$ & 256489 & 448 & 10 \\
\hline 62 & 01a_11a & 127033 & 630 & 22 \\
\hline 63 & 11a_21a & 175045 & 568 & 18 \\
\hline 64 & 21a_31a & 184213 & 574 & 18 \\
\hline 65 & 31a_01a & 127733 & 636 & 22 \\
\hline
\end{tabular}


Table A.2. Kinematic structures found in region 00 in the $U-V$ (Plane 1), $V-\sqrt{U^{2}+2 V^{2}}$ (Plane 2), $L_{z}-\sqrt{L_{x}^{2}+L_{y}^{2}}$ (Plane 3) and $L_{z}-\sqrt{J_{\mathrm{r}}}$ (Plane 4) planes at scale $J=2$.

\begin{tabular}{|c|c|c|c|c|c|c|c|c|c|c|c|c|}
\hline$N$ & Plane & Group & Name & $N$ stars & $\begin{array}{r}U \\
{\left[\mathrm{~km} \mathrm{~s}^{-1}\right]} \\
\end{array}$ & $\begin{array}{r}V \\
{\left[\mathrm{~km} \mathrm{~s}^{-1}\right]} \\
\end{array}$ & $\begin{array}{r}L_{z} \\
{\left[\mathrm{kpc} \mathrm{km} \mathrm{s}^{-1}\right]} \\
\end{array}$ & $\begin{array}{r}\sqrt{J_{\mathrm{r}}} \\
{\left[\mathrm{kpc} \mathrm{km} \mathrm{s}^{-1}\right]} \\
\end{array}$ & $\begin{array}{r}\sigma_{U} \\
{\left[\mathrm{~km} \mathrm{~s}^{-1}\right]} \\
\end{array}$ & $\begin{array}{r}\sigma_{V} \\
{\left[\mathrm{~km} \mathrm{~s}^{-1}\right]} \\
\end{array}$ & $\begin{array}{r}\sigma_{L_{z}} \\
{\left[\mathrm{kpc} \mathrm{km} \mathrm{s}^{-1}\right]} \\
\end{array}$ & $\begin{array}{r}\sigma \sqrt{J_{\mathrm{r}}} \\
{\left[\mathrm{kpc} \mathrm{km} \mathrm{s}^{-1}\right]} \\
\end{array}$ \\
\hline 1 & 1 & g1 & $\mathrm{A} 1 / \mathrm{A} 2$ & 173 & 39 & 37 & 2158 & 12 & 2 & 2 & 30 & 0.6 \\
\hline 2 & 1 & $\mathrm{~g} 2$ & $\gamma$ Leo & 2643 & 34 & 8 & 1926 & 7 & 2 & 2 & 28 & 0.5 \\
\hline 3 & 1 & g3 & Sirius & 6966 & -13 & 6 & 1918 & 3.6 & 2 & 2 & 31 & 0.5 \\
\hline 4 & 1 & $\mathrm{~g} 4$ & Sirius & 1659 & -53 & 4 & 1900 & 6.3 & 2 & 2 & 30 & 0.5 \\
\hline 5 & 1 & g5 & $\gamma$ Leo & 2277 & 47 & 3 & 1894 & 7.9 & 2 & 2 & 31 & 0.5 \\
\hline 6 & 1 & g6 & Sirius & 10303 & 12 & 2 & 1888 & 4.1 & 2 & 2 & 30 & 0.5 \\
\hline 7 & 1 & g7 & Sirius & 1416 & -61 & 2 & 1887 & 6.9 & 2 & 2 & 30 & 0.5 \\
\hline 8 & 1 & g8 & $\gamma$ Leo & 1080 & 65 & 1 & 1872 & 9.9 & 2 & 2 & 27 & 0.5 \\
\hline 9 & 1 & g9 & Sirius & 871 & -79 & 0 & 1858 & 8.8 & 2 & 2 & 31 & 0.5 \\
\hline 10 & 1 & $\mathrm{~g} 10$ & $\gamma$ Leo & 785 & 73 & -2 & 1844 & 10.6 & 2 & 2 & 28 & 0.5 \\
\hline 11 & 1 & $\mathrm{~g} 11$ & $\gamma$ Leo & 1881 & 57 & -5 & 1819 & 8.5 & 2 & 2 & 32 & 0.5 \\
\hline 12 & 1 & $\mathrm{~g} 12$ & Coma Berenices & 12046 & -15 & -7 & 1808 & 1.3 & 2 & 2 & 29 & 0.5 \\
\hline 13 & 1 & $\mathrm{~g} 13$ & Bobylev16 & 180 & -118 & -10 & 1776 & 13.1 & 2 & 2 & 31 & 0.5 \\
\hline 14 & 1 & g14 & Coma Berenices & 8439 & 8 & -14 & 1745 & 2.4 & 2 & 2 & 30 & 0.5 \\
\hline 15 & 1 & $\mathrm{~g} 15$ & Pleiades/Hyades & 960 & -81 & -14 & 1744 & 8.3 & 2 & 2 & 29 & 0.5 \\
\hline 16 & 1 & $\mathrm{~g} 16$ & Pleiades/Hyades & 14380 & -33 & -16 & 1733 & 2.7 & 2 & 2 & 28 & 0.5 \\
\hline 17 & 1 & $\mathrm{~g} 17$ & Pleiades/Hyades & 11749 & -42 & -19 & 1710 & 3.8 & 2 & 2 & 25 & 0.4 \\
\hline 18 & 1 & $\mathrm{~g} 18$ & Antoja12(12) & 302 & 98 & -21 & 1695 & 13 & 2 & 2 & 29 & 0.5 \\
\hline 19 & 1 & g19 & Wolf 630 & 6104 & 21 & -22 & 1678 & 4.1 & 2 & 2 & 29 & 0.5 \\
\hline 20 & 1 & $\mathrm{~g} 20$ & Dehnen98 & 4129 & 41 & -25 & 1664 & 6.3 & 2 & 2 & 26 & 0.4 \\
\hline 21 & 1 & $\mathrm{~g} 21$ & Pleiades/Hyades & 13084 & -10 & -25 & 1657 & 2.2 & 2 & 2 & 26 & 0.4 \\
\hline 22 & 1 & $\mathrm{~g} 22$ & Hercules & 2604 & -58 & -37 & 1562 & 6.5 & 2 & 2 & 24 & 0.4 \\
\hline 23 & 1 & $\mathrm{~g} 23$ & Pleiades/Hyades & 3428 & 25 & -37 & 1561 & 5.7 & 2 & 2 & 27 & 0.4 \\
\hline 24 & 1 & $\mathrm{~g} 24$ & Hercules & 1380 & -82 & -47 & 1473 & 9.5 & 2 & 2 & 27 & 0.4 \\
\hline 25 & 1 & g25 & Hercules & 3529 & -15 & -48 & 1468 & 5.8 & 2 & 2 & 25 & 0.4 \\
\hline 26 & 1 & g26 & Hercules & 4626 & -37 & -49 & 1463 & 6.6 & 2 & 2 & 22 & 0.4 \\
\hline 27 & 1 & $\mathrm{~g} 27$ & Hercules & 2816 & 0 & -50 & 1455 & 6.2 & 2 & 2 & 24 & 0.5 \\
\hline 28 & 1 & $\mathrm{~g} 28$ & Hercules & 1593 & -70 & -51 & 1450 & 8.9 & 2 & 2 & 25 & 0.5 \\
\hline 29 & 1 & $\mathrm{~g} 29$ & Antoja12(15) & 633 & 65 & -52 & 1441 & 10.2 & 2 & 2 & 27 & 0.4 \\
\hline 30 & 1 & g30 & HR1614 & 1286 & 9 & -64 & 1342 & 8.2 & 2 & 2 & 25 & 0.5 \\
\hline 31 & 1 & $\mathrm{~g} 31$ & HR1614 & 1989 & -16 & -64 & 1343 & 8.1 & 2 & 2 & 25 & 0.4 \\
\hline 32 & 1 & g32 & HR1614 & 1619 & -26 & -67 & 1321 & 8.5 & 2 & 2 & 24 & 0.5 \\
\hline 33 & 1 & $\mathrm{~g} 33$ & HR1614 & 1345 & -36 & -68 & 1313 & 8.9 & 2 & 2 & 25 & 0.5 \\
\hline 34 & 1 & g34 & $\epsilon$ Ind & 498 & -83 & -75 & 1252 & 12 & 2 & 2 & 25 & 0.4 \\
\hline 35 & 1 & g35 & $\epsilon$ Ind & 626 & -73 & -76 & 1247 & 11.5 & 2 & 2 & 24 & 0.5 \\
\hline 36 & 1 & g36 & Arcturus & 358 & -13 & -92 & 1118 & 11.6 & 2 & 2 & 23 & 0.6 \\
\hline 37 & 2 & $\mathrm{~g} 1$ & Arcturus & 1840 & -11 & -91 & 1125 & 11.7 & 25 & 2 & 22 & 0.6 \\
\hline 38 & 2 & $\mathrm{~g} 2$ & AF06 & 2013 & -12 & -86 & 1168 & 11.1 & 26 & 2 & 24 & 0.7 \\
\hline 39 & 2 & g3 & Hercules & 1483 & -85 & -54 & 1431 & 10.4 & 57 & 2 & 26 & 0.8 \\
\hline 40 & 2 & g4 & HR1614 & 7629 & -10 & -65 & 1337 & 8.3 & 20 & 1 & 23 & 0.5 \\
\hline 41 & 2 & g5 & HR1614 & 8490 & -9 & -61 & 1366 & 7.8 & 19 & 1 & 24 & 0.5 \\
\hline 42 & 2 & g6 & Hercules & 14860 & -10 & -46 & 1482 & 5.8 & 19 & 2 & 27 & 0.5 \\
\hline 43 & 2 & g7 & Sirius & 2459 & -60 & 1 & 1876 & 8 & 64 & 2 & 29 & 1.4 \\
\hline 44 & 2 & g8 & Hercules & 15404 & -5 & -40 & 1539 & 4.9 & 21 & 2 & 25 & 0.7 \\
\hline 45 & 2 & g9 & $\mathrm{A} 1 / \mathrm{A} 2$ & 1129 & -5 & 38 & 2173 & 10.6 & 17 & 2 & 30 & 0.6 \\
\hline 46 & 2 & $\mathrm{~g} 10$ & Pleiades/Hyades & 16788 & -41 & -19 & 1707 & 3.9 & 34 & 2 & 26 & 1.1 \\
\hline 47 & 2 & g11 & $\gamma$ Leo & 4112 & 45 & 3 & 1893 & 7.4 & 49 & 2 & 31 & 1.3 \\
\hline 48 & 2 & $\mathrm{~g} 12$ & Pleiades/Hyades & 38181 & -7 & -25 & 1660 & 2.5 & 14 & 2 & 27 & 0.7 \\
\hline 49 & 2 & $\mathrm{~g} 13$ & $\gamma$ Leo & 5460 & 30 & 7 & 1921 & 6.3 & 34 & 2 & 29 & 1.1 \\
\hline 50 & 2 & $\mathrm{~g} 14$ & $\mathrm{~A} 1 / \mathrm{A} 2$ & 3361 & -5 & 22 & 2044 & 7 & 13 & 1 & 32 & 0.6 \\
\hline 51 & 2 & $\mathrm{~g} 15$ & Coma Berenices & 26737 & -2 & -12 & 1766 & 1.2 & 10 & 2 & 29 & 1 \\
\hline 52 & 2 & $\mathrm{~g} 16$ & Sirius & 19311 & 0 & 8 & 1926 & 4.3 & 9 & 2 & 27 & 0.5 \\
\hline 53 & 2 & $\mathrm{~g} 17$ & Sirius & 19358 & 0 & 0 & 1872 & 2.9 & 5 & 2 & 31 & 0.5 \\
\hline
\end{tabular}

Notes. First column is a line number in the table; the second one denotes the plane; names of the groups as in Fig. 3 are given in Col. 3 and names of the groups as in the literature are provided in Col. 4; the number of stars in each group is given in Col. 5; median $U, V$ velocities, median angular momentum $L_{z}$ and the median value of the square root of radial action per group is given in Cols. 6-9; Cols. 8-13 are standard deviations of the same quantities as in Cols. 6-9. 
Table A.2. continued.

\begin{tabular}{|c|c|c|c|c|c|c|c|c|c|c|c|c|}
\hline$N$ & Plane & Group & Name & $N$ stars & $\begin{array}{r}U \\
{\left[\mathrm{~km} \mathrm{~s}^{-1}\right]} \\
\end{array}$ & $\begin{array}{r}V \\
{\left[\mathrm{~km} \mathrm{~s}^{-1}\right]} \\
\end{array}$ & $\begin{array}{r}L_{z} \\
{\left[\mathrm{kpc} \mathrm{km} \mathrm{s}^{-1}\right]} \\
\end{array}$ & $\begin{array}{r}\sqrt{J_{\mathrm{r}}} \\
{\left[\mathrm{kpc} \mathrm{km} \mathrm{s}^{-1}\right]} \\
\end{array}$ & $\begin{array}{r}\sigma_{U} \\
{\left[\mathrm{~km} \mathrm{~s}^{-1}\right]} \\
\end{array}$ & $\begin{array}{r}\sigma_{V} \\
{\left[\mathrm{~km} \mathrm{~s}^{-1}\right]} \\
\end{array}$ & $\begin{array}{r}\sigma_{L_{z}} \\
{\left[\mathrm{kpc} \mathrm{km} \mathrm{s}^{-1}\right]} \\
\end{array}$ & $\begin{array}{r}\sigma \sqrt{J_{\mathrm{r}}} \\
{\left[\mathrm{kpc} \mathrm{km} \mathrm{s}^{-1}\right]}\end{array}$ \\
\hline 54 & 3 & g1 & Pleiades/Hyades & 81 & -13 & -23 & 1661 & 3.2 & 40 & 2 & 2 & 2.8 \\
\hline 55 & 3 & g2 & Hercules & 47 & -12 & -52 & 1439 & 6.6 & 47 & 2 & 2 & 2.3 \\
\hline 56 & 3 & g3 & Hercules & 66 & -18 & -41 & 1529 & 5.7 & 47 & 2 & 2 & 2.3 \\
\hline 57 & 3 & g4 & Sirius & 86 & -6 & 4 & 1895 & 4.4 & 33 & 3 & 2 & 1.9 \\
\hline 58 & 3 & g5 & HR1614 & 41 & -17 & -67 & 1325 & 8.7 & 45 & 2 & 1 & 1.9 \\
\hline 59 & 3 & g6 & Pleiades/Hyades & 171 & -12 & -20 & 1698 & 3.5 & 34 & 2 & 2 & 2.1 \\
\hline 60 & 3 & g7 & Hercules & 120 & -27 & -51 & 1452 & 6.7 & 34 & 2 & 2 & 1.3 \\
\hline 61 & 3 & g8 & Sirius & 165 & -3 & 2 & 1890 & 4.2 & 35 & 3 & 2 & 2.2 \\
\hline 62 & 3 & g9 & Pleiades/Hyades & 264 & -19 & -20 & 1698 & 2.8 & 36 & 2 & 2 & 2.5 \\
\hline 63 & 3 & g10 & AF06 & 17 & -22 & -94 & 1109 & 12.4 & 45 & 2 & 2 & 1.5 \\
\hline 64 & 3 & g11 & $\mathrm{A} 1 / \mathrm{A} 2$ & 41 & -10 & 24 & 2064 & 8.1 & 38 & 3 & 2 & 2 \\
\hline 65 & 3 & $\mathrm{~g} 12$ & Sirius & 263 & -2 & 7 & 1926 & 4.6 & 29 & 3 & 2 & 1.7 \\
\hline 66 & 3 & g13 & $\mathrm{A} 1 / \mathrm{A} 2$ & 55 & -13 & 26 & 2063 & 7.9 & 30 & 3 & 2 & 1.5 \\
\hline 67 & 3 & g14 & HR1614 & 119 & -11 & -62 & 1350 & 8.4 & 38 & 2 & 2 & 1.3 \\
\hline 68 & 3 & g15 & Hercules & 339 & -20 & -39 & 1547 & 5.4 & 39 & 2 & 2 & 1.7 \\
\hline 69 & 3 & $\mathrm{~g} 16$ & Sirius & 456 & 0 & 6 & 1914 & 4.3 & 28 & 3 & 2 & 1.7 \\
\hline 70 & 3 & g17 & $\mathrm{A} 1 / \mathrm{A} 2$ & 54 & -16 & 24 & 2057 & 7.5 & 28 & 3 & 2 & 1.5 \\
\hline 71 & 3 & g18 & HR1614 & 108 & -17 & -62 & 1349 & 8.4 & 39 & 2 & 2 & 1.6 \\
\hline 72 & 3 & g19 & Hercules & 379 & -32 & -48 & 1470 & 6.5 & 31 & 2 & 2 & 1.4 \\
\hline 73 & 3 & g20 & Sirius & 427 & 3 & 5 & 1919 & 4.2 & 23 & 3 & 2 & 1.5 \\
\hline 74 & 3 & g21 & Arcturus & 8 & -44 & -111 & 967 & 15.4 & 60 & 1 & 3 & 0.6 \\
\hline 75 & 3 & g22 & Coma Berenices & 644 & -9 & -7 & 1806 & 2 & 27 & 2 & 2 & 2.3 \\
\hline 76 & 3 & g23 & Pleiades/Hyades & 865 & -21 & -19 & 1703 & 2.5 & 25 & 2 & 2 & 1.7 \\
\hline 77 & 3 & $\mathrm{~g} 24$ & HR1614 & 90 & -18 & -61 & 1362 & 8.3 & 39 & 2 & 2 & 1.5 \\
\hline 78 & 4 & $\mathrm{~g} 1$ & KFR08 & 107 & -13 & -160 & 575 & 19.5 & 49 & 2 & 23 & 0.2 \\
\hline 79 & 4 & $\mathrm{~g} 2$ & Arcturus & 303 & -6 & -127 & 841 & 16.1 & 40 & 3 & 23 & 0.2 \\
\hline 80 & 4 & g3 & Antoja12(12) & 122 & 103 & -19 & 1707 & 15.1 & 125 & 3 & 22 & 0.3 \\
\hline 81 & 4 & g4 & $\mathrm{A} 1 / \mathrm{A} 2$ & 132 & 31 & 43 & 2223 & 13.3 & 42 & 4 & 22 & 0.2 \\
\hline 82 & 4 & g5 & Hercules & 326 & -111 & -50 & 1460 & 13 & 103 & 3 & 22 & 0.2 \\
\hline 83 & 4 & g6 & $\mathrm{A} 1 / \mathrm{A} 2$ & 255 & 27 & 30 & 2110 & 12.8 & 69 & 4 & 22 & 0.2 \\
\hline 84 & 4 & g7 & Antoja12(12) & 579 & 92 & -19 & 1710 & 12.9 & 105 & 4 & 23 & 0.3 \\
\hline 85 & 4 & g8 & $\gamma$ Leo & 405 & 84 & -7 & 1805 & 12.7 & 102 & 4 & 22 & 0.2 \\
\hline 86 & 4 & g9 & AF06 & 1836 & -18 & -92 & 1113 & 11.9 & 27 & 2 & 21 & 0.2 \\
\hline 87 & 4 & g10 & $\gamma$ Leo & 803 & 77 & -4 & 1828 & 11.4 & 84 & 4 & 23 & 0.3 \\
\hline 88 & 4 & g11 & $\mathrm{A} 1 / \mathrm{A} 2$ & 1026 & -7 & 39 & 2177 & 10.9 & 26 & 3 & 20 & 0.3 \\
\hline 89 & 4 & $\mathrm{~g} 12$ & $\gamma$ Leo & 1765 & 64 & 0 & 1867 & 10 & 70 & 4 & 23 & 0.3 \\
\hline 90 & 4 & $\mathrm{~g} 13$ & Hercules & 3649 & -72 & -50 & 1460 & 9.4 & 64 & 4 & 23 & 0.3 \\
\hline 91 & 4 & g14 & $\gamma$ Leo & 3393 & 48 & 1 & 1878 & 8.3 & 57 & 4 & 24 & 0.2 \\
\hline 92 & 4 & $\mathrm{~g} 15$ & HR1614 & 8691 & -15 & -64 & 1342 & 8.3 & 22 & 2 & 21 & 0.3 \\
\hline 93 & 4 & $\mathrm{~g} 16$ & $\mathrm{~A} 1 / \mathrm{A} 2$ & 2922 & -13 & 25 & 2064 & 7.7 & 20 & 3 & 20 & 0.3 \\
\hline 94 & 4 & g17 & $\gamma$ Leo & 5271 & 33 & 6 & 1910 & 7 & 44 & 4 & 23 & 0.3 \\
\hline 95 & 4 & $\mathrm{~g} 18$ & Hercules & 16701 & -21 & -49 & 1463 & 6.2 & 19 & 2 & 20 & 0.3 \\
\hline 96 & 4 & $\mathrm{~g} 19$ & Dehnen98 & 7245 & 36 & -23 & 1679 & 6.1 & 47 & 4 & 22 & 0.3 \\
\hline 97 & 4 & $\mathrm{~g} 20$ & Pleiades/Hyades & 15246 & -15 & -38 & 1551 & 4.6 & 19 & 3 & 22 & 0.3 \\
\hline 98 & 4 & $\mathrm{~g} 21$ & Sirius & 22970 & 0 & 6 & 1916 & 4.1 & 15 & 3 & 22 & 0.3 \\
\hline 99 & 4 & g22 & Pleiades/Hyades & 21418 & -39 & -20 & 1699 & 3.9 & 29 & 4 & 22 & 0.3 \\
\hline 100 & 4 & $\mathrm{~g} 23$ & Pleiades/Hyades & 32987 & -13 & -25 & 1664 & 2.4 & 11 & 3 & 22 & 0.2 \\
\hline 101 & 4 & g24 & Coma Berenices & 21624 & -11 & -11 & 1769 & 0.9 & 6 & 3 & 23 & 0.2 \\
\hline
\end{tabular}


Table A.3. Kinematic structures found in region 00 in the $U-V$ (Plane 1), $V-\sqrt{U^{2}+2 V^{2}}$ (Plane 2), $L_{z}-\sqrt{L_{x}^{2}+L_{y}^{2}}$ (Plane 3 ) and $L_{z}-\sqrt{J_{\mathrm{r}}}$ (Plane 4) planes at scale $J=3$.

\begin{tabular}{|c|c|c|c|c|c|c|c|c|c|c|c|c|}
\hline$N$ & Plane & Group & Name & $N$ stars & $\begin{array}{r}U \\
{\left[\mathrm{~km} \mathrm{~s}^{-1}\right]} \\
\end{array}$ & $\begin{array}{r}V \\
{\left[\mathrm{~km} \mathrm{~s}^{-1}\right]} \\
\end{array}$ & $\begin{array}{r}L_{z} \\
{\left[\mathrm{kpc} \mathrm{km} \mathrm{s}^{-1}\right]} \\
\end{array}$ & $\begin{array}{r}\sqrt{J_{\mathrm{r}}} \\
{\left[\mathrm{kpc} \mathrm{km} \mathrm{s}^{-1}\right]} \\
\end{array}$ & $\begin{array}{r}\sigma_{U} \\
{\left[\mathrm{~km} \mathrm{~s}^{-1}\right]} \\
\end{array}$ & $\begin{array}{r}\sigma_{V} \\
{\left[\mathrm{~km} \mathrm{~s}^{-1}\right]} \\
\end{array}$ & $\begin{array}{r}\sigma_{L_{z}} \\
{\left[\mathrm{kpc} \mathrm{km} \mathrm{s}^{-1}\right]} \\
\end{array}$ & $\begin{array}{r}\sigma \sqrt{J_{\mathrm{r}}} \\
{\left[\mathrm{kpc} \mathrm{km} \mathrm{s}^{-1}\right]}\end{array}$ \\
\hline 1 & 1 & g1 & $\mathrm{A} 1 / \mathrm{A} 2$ & 44 & -122 & 17 & 2003 & 15.9 & 2 & 2 & 34 & 0.5 \\
\hline 2 & 1 & g2 & Sirius & 10806 & 9 & 2 & 1890 & 3.9 & 2 & 2 & 30 & 0.5 \\
\hline 3 & 1 & g3 & $\gamma$ Leo & 2325 & 49 & 0 & 1872 & 7.9 & 2 & 2 & 32 & 0.5 \\
\hline 4 & 1 & g4 & Bobylev16 & 824 & -83 & -3 & 1840 & 9.1 & 2 & 2 & 30 & 0.5 \\
\hline 5 & 1 & g5 & Coma Berenices & 12027 & -11 & -6 & 1814 & 1.3 & 2 & 2 & 29 & 0.5 \\
\hline 6 & 1 & g6 & Bobylev16 & 194 & -118 & -13 & 1750 & 12.9 & 2 & 2 & 29 & 0.4 \\
\hline 7 & 1 & g7 & Pleiades/Hyades & 14437 & -33 & -17 & 1726 & 2.7 & 2 & 2 & 28 & 0.5 \\
\hline 8 & 1 & g8 & Antoja12(12) & 332 & 96 & -22 & 1686 & 12.8 & 2 & 2 & 30 & 0.5 \\
\hline 9 & 1 & g9 & Pleiades/Hyades & 14069 & -14 & -23 & 1672 & 2 & 2 & 2 & 28 & 0.4 \\
\hline 10 & 1 & $\mathrm{~g} 10$ & Wolf 630 & 5913 & 21 & -24 & 1665 & 4.2 & 2 & 2 & 28 & 0.5 \\
\hline 11 & 1 & g11 & Dehnen98 & 4086 & 41 & -25 & 1659 & 6.4 & 2 & 2 & 26 & 0.5 \\
\hline 12 & 1 & g12 & Hercules & 4670 & -37 & -49 & 1463 & 6.5 & 2 & 2 & 22 & 0.4 \\
\hline 13 & 1 & g13 & Hercules & 1394 & -81 & -49 & 1464 & 9.6 & 2 & 2 & 25 & 0.4 \\
\hline 14 & 1 & g14 & Hercules & 2400 & 1 & -53 & 1438 & 6.5 & 2 & 2 & 26 & 0.5 \\
\hline 15 & 1 & g15 & HR1614 & 1769 & -13 & -66 & 1331 & 8.2 & 2 & 2 & 25 & 0.5 \\
\hline 16 & 1 & g16 & $\epsilon$ Ind & 544 & -76 & -77 & 1239 & 11.7 & 2 & 2 & 23 & 0.5 \\
\hline 17 & 2 & $\mathrm{~g} 1$ & Arcturus & 1837 & -13 & -92 & 1113 & 11.9 & 28 & 2 & 22 & 0.7 \\
\hline 18 & 2 & g2 & HR1614 & 7724 & -14 & -66 & 1330 & 8.5 & 24 & 2 & 24 & 0.5 \\
\hline 19 & 2 & g3 & Hercules & 15998 & -20 & -49 & 1463 & 6.3 & 24 & 2 & 25 & 0.5 \\
\hline 20 & 2 & g4 & Hercules & 15702 & -12 & -42 & 1524 & 5.4 & 25 & 2 & 29 & 0.7 \\
\hline 21 & 2 & g5 & $\mathrm{A} 1 / \mathrm{A} 2$ & 1270 & -6 & 37 & 2167 & 10.5 & 21 & 2 & 32 & 0.6 \\
\hline 22 & 2 & g6 & $\mathrm{A} 1 / \mathrm{A} 2$ & 1835 & -7 & 30 & 2108 & 8.8 & 17 & 2 & 36 & 0.6 \\
\hline 23 & 2 & g7 & Pleiades/Hyades & 40542 & -11 & -21 & 1696 & 2 & 17 & 2 & 29 & 0.9 \\
\hline 24 & 2 & g8 & Sirius & 21306 & 6 & 0 & 1865 & 3 & 10 & 2 & 33 & 0.8 \\
\hline 25 & 3 & g1 & HR1614 & 35 & -16 & -68 & 1315 & 8.7 & 40 & 2 & 2 & 1.7 \\
\hline 26 & 3 & g2 & Pleiades/Hyades & 190 & -11 & -21 & 1692 & 3.3 & 39 & 2 & 2 & 2.6 \\
\hline 27 & 3 & g3 & Hercules & 125 & -26 & -50 & 1457 & 6.6 & 42 & 2 & 2 & 1.8 \\
\hline 28 & 3 & g4 & Sirius & 165 & -3 & 2 & 1890 & 4.2 & 35 & 3 & 2 & 2.2 \\
\hline 29 & 3 & g5 & HR1614 & 85 & -33 & -68 & 1308 & 9.2 & 37 & 2 & 2 & 1.5 \\
\hline 30 & 3 & g6 & Sirius & 522 & 6 & 4 & 1901 & 4.1 & 27 & 3 & 2 & 1.7 \\
\hline 31 & 3 & g7 & Hercules & 383 & -31 & -49 & 1464 & 6.6 & 33 & 2 & 2 & 1.4 \\
\hline 32 & 3 & g8 & Pleiades/Hyades & 939 & -20 & -20 & 1697 & 2.8 & 28 & 2 & 2 & 1.8 \\
\hline 33 & 3 & g9 & Arcturus & 8 & -49 & -106 & 1000 & 14.2 & 38 & 1 & 2 & 0.8 \\
\hline 34 & 4 & g1 & KFR08 & 94 & -14 & -159 & 586 & 19.3 & 51 & 2 & 21 & 0.2 \\
\hline 35 & 4 & $\mathrm{~g} 2$ & Antoja12(12) & 585 & 91 & -18 & 1718 & 12.8 & 104 & 4 & 23 & 0.3 \\
\hline 36 & 4 & g3 & $\mathrm{A} 1 / \mathrm{A} 2$ & 1081 & -6 & 38 & 2172 & 10.8 & 27 & 3 & 22 & 0.3 \\
\hline 37 & 4 & g4 & Hercules & 2939 & -77 & -49 & 1463 & 9.7 & 70 & 4 & 23 & 0.2 \\
\hline 38 & 4 & g5 & $\gamma$ Leo & 3455 & 49 & 0 & 1869 & 8.3 & 58 & 4 & 24 & 0.2 \\
\hline 39 & 4 & g6 & $\mathrm{A} 1 / \mathrm{A} 2$ & 3076 & -14 & 24 & 2058 & 7.6 & 21 & 3 & 21 & 0.3 \\
\hline 40 & 4 & g7 & $\gamma$ Leo & 4959 & 35 & 6 & 1910 & 7.2 & 45 & 4 & 24 & 0.2 \\
\hline 41 & 4 & g8 & Hercules & 17305 & -24 & -49 & 1466 & 6.3 & 21 & 3 & 21 & 0.3 \\
\hline 42 & 4 & g9 & Sirius & 23880 & 0 & 5 & 1909 & 4 & 15 & 3 & 22 & 0.3 \\
\hline 43 & 4 & g10 & Pleiades/Hyades & 35424 & -17 & -22 & 1687 & 2.3 & 13 & 3 & 24 & 0.2 \\
\hline
\end{tabular}

Notes. First column is a line number in the table; the second one denotes the plane; names of the groups as in Fig. 4 are given in Col. 3 and names of the groups as in the literature are provided in column 4; number of stars in each group is given in Col. 5; median $U, V$ velocities, median angular momentum $L_{z}$ and the median value of the square root of radial action per group is given in Cols. 6-9; Cols. 8-13 are standard deviations of the same quantities as in Cols. 6-9. 